\title{
Diamond Magnetic Microscopy of Malarial Hemozoin Nanocrystals
}

\author{
Ilja Fescenko,,${ }^{1, *}$ Abdelghani Laraoui, ${ }^{1}$ Janis Smits, ${ }^{1,2}$ Nazanin Mosavian, ${ }^{1}$ Pauli Kehayias, ${ }^{1,3}$ \\ Jong Seto, ${ }^{4}$ Lykourgos Bougas, ${ }^{5}$ Andrey Jarmola, ${ }^{6,7}$ and Victor M. Acosta ${ }^{1, \dagger}$ \\ ${ }^{1}$ Center for High Technology Materials and Department of Physics and Astronomy, University of New Mexico, \\ 1313 Goddard St SE, Albuquerque, 87106 New Mexico, USA \\ ${ }^{2}$ Laser Centre of the University of Latvia, Jelgavas street 3, Riga, LV-1004, Latvia \\ ${ }^{3}$ Department of Physics, Harvard University, 17 Oxford St, Cambridge, 02138 Massachusetts, USA \\ ${ }^{4}$ Department of Bioengineering and Therapeutic Sciences, School of Medicine, University of \\ California-San Francisco, 1700 4th St, San Francisco, 94158 California, USA \\ ${ }^{5}$ Johannes Guttenberg University, Saarstraße 21, 55128 Mainz, Germany \\ ${ }^{6}$ Department of Physics, University of California, Berkeley, 366 LeConte Hall, Berkeley, 94720 California, USA \\ ${ }^{7}$ ODMR Technologies Inc., 2041 Tapscott Ave, El Cerrito, 94530 California, USA
}

(Received 3 October 2018; revised manuscript received 23 December 2018; published 12 March 2019)

\begin{abstract}
Magnetic microscopy of malarial hemozoin nanocrystals is performed by optically detected magnetic resonance imaging of near-surface diamond nitrogen-vacancy centers. Hemozoin crystals are extracted from Plasmodium falciparum -infected human blood cells and studied alongside synthetic hemozoin crystals. The stray magnetic fields produced by individual crystals are imaged at room temperature as a function of the applied field up to $350 \mathrm{mT}$. More than 100 nanocrystals are analyzed, revealing the distribution of their magnetic properties. Most crystals (96\%) exhibit a linear dependence of the strayfield magnitude on the applied field, confirming hemozoin's paramagnetic nature. A volume magnetic susceptibility of $3.4 \times 10^{-4}$ is inferred with use of a magnetostatic model informed by correlated scanning-electron-microscopy measurements of crystal dimensions. A small fraction of nanoparticles (4/82 for Plasmodium falciparum-produced nanoparticles and 1/41 for synthetic nanoparticles) exhibit a saturation behavior consistent with superparamagnetism. Translation of this platform to the study of living Plasmodium-infected cells may shed new light on hemozoin formation dynamics and their interaction with antimalarial drugs.
\end{abstract}

DOI: 10.1103/PhysRevApplied.11.034029

\section{INTRODUCTION}

Magnetic field sensors based on diamond nitrogenvacancy (NV) centers have emerged as a powerful platform for detecting nanomagnetism in biological samples [1,2]. With this technique, magnetic fields from magnetotactic bacteria [3], ferritin proteins [4], magnetically labeled cancer cells [5], and neuronal currents [6] have been detected with a remarkable combination of spatial resolution and sensitivity. Diamond magnetic microscopy has even been able to resolve magnetic fields produced by individual nanoparticles exhibiting

\footnotetext{
*iliafes@gmail.com

†victormarcelacosta@gmail.com
}

Published by the American Physical Society under the terms of the Creative Commons Attribution 4.0 International license. Further distribution of this work must maintain attribution to the author(s) and the published article's title, journal citation, and DOI. ferromagnetism and superparamagnetism [7-9]. However, observation of individual paramagnetic nanoparticles at ambient temperature has remained a challenge, owing to their weaker magnetic signatures.

Of particular interest are paramagnetic hemozoin biocrystals that nucleate inside several blood-feeding organisms [10], including the Plasmodium species responsible for malaria. Malarial parasites feed on their host's hemoglobin for essential amino acids, while decomposing the iron complexes into highly toxic free radicals. These radicals are subsequently bound into chemically inert elongated crystals (50-1500 nm in size) called "hemozoin" [10-12]. Hemozoin crystals are a biomarker for malaria, and a substantial effort has been devoted to developing diagnostic platforms based on their detection $[10,13]$. Hemozoin detection is also used in pharmacological studies of malaria [14], since some antimalarial drugs work by altering hemozoin formation $[11,12]$.

Hemozoin crystals have characteristic optical properties, including birefringence [15], linear dichroism [16], 
and nonlinear dielectric susceptibility [17], that allow their detection without the use of extrinsic labels. They are also paramagnetic due to the presence of unpaired electrons in their $\mathrm{Fe}^{3+}$ centers, meaning they are magnetized only in the presence of an external magnetic field. Direct detection of hemozoin's magnetization is intriguing because it gives quantitative information related to crystal size and iron composition.

Several methods of studying hemozoin magnetic signatures have been demonstrated, including magneto-optical rotation [18-20], nuclear-magnetic-resonance relaxometry [21,22], electron paramagnetic resonance [23], and magnetic separation [24]. Direct magnetic detection of hemozoin ensembles has been performed by bulk magnetometry [25-27]. However, detection of stray magnetic fields produced by single hemozoin nanocrystals has not yet been reported, likely due to stringent requirements on sensitivity and spatial resolution.

We hypothesized that diamond magnetic microscopy $[3,5,9,28,29]$ possesses sufficient sensitivity and spatial resolution to image the stray magnetic field produced by individual hemozoin nanocrystals. To test this, we performed room-temperature optically-detected-magneticresonance (ODMR) imaging of a NV-doped diamond substrate in contact with either "natural" (Plasmodiumproduced) or synthetic hemozoin nanocrystals. Spatially resolved maps of the magnetic fields produced by individual hemozoin nanocrystals were obtained and used to characterize the distribution of their paramagnetic properties. With appropriate modifications, this detection strategy may be used to study the formation dynamics of hemozoin crystals in living Plasmodium-infected cells.

\section{DETECTION PRINCIPLE}

NV centers are spin- 1 defects in the diamond lattice. The energy levels and optical excitation and emission pathways of the NV center are shown in Fig. 1(a). For a magnetic field $B_{\|}$applied along the NV symmetry axis, the $|0\rangle \leftrightarrow| \pm 1\rangle$ spin transition frequencies are $f_{ \pm}=D \pm$ $\gamma_{\mathrm{NV}} B_{\|}$, where $D=2.87 \mathrm{GHz}$ is the zero-field splitting and $\gamma_{\mathrm{NV}}=28 \mathrm{GHz} / \mathrm{T}$ is the NV gyromagnetic ratio [Fig. 1(b)]. The principle of NV magnetometry is to measure these transition frequencies precisely with ODMR techniques $[1,2]$. When 532-nm light continuously excites NV centers, the ground-state population is optically polarized into $|0\rangle$ via a nonradiative, spin-selective decay pathway involving intermediate singlet states. Because of the same spin-selective decay mechanism, NV centers excited from $|0\rangle$ emit fluorescence (collected at $650-800 \mathrm{~nm}$ ) at a higher rate than those originating from $| \pm 1\rangle$. Application of a transverse microwave magnetic field mixes the spin populations, resulting in a dip in fluorescence when the microwave frequency matches the spin transition frequencies, $f_{ \pm}$[Fig. 1(c)]. Monitoring of NV fluorescence as the microwave frequency is swept across the resonances reveals $f_{ \pm}$and thus $B_{\|}$.

The minimum detectable magnetic field of a Lorentzian ODMR signal is limited by photoelectron shot noise as $[2,30,31]$

$$
B_{\min } \simeq \frac{4}{3 \sqrt{3}} \frac{\Gamma}{\gamma_{\mathrm{NV}} C \sqrt{I_{0} t}}
$$

where $\Gamma$ is the full-width-at-half-maximum (FWHM) linewidth, $C$ is the contrast (the relative difference in ODMR signal on and off resonance), $I_{0}$ is the photoelectron detection rate, and $t$ is the measurement time. In room-temperature NV ODMR experiments, the spin projection noise is typically more than one order of magnitude lower than the photoelectron shot noise and is therefore negligible [32-34]. With typical experimental values $\left(\Gamma=12 \mathrm{MHz}, C=0.02, I_{0}=5 \times 10^{6}\right.$ electrons $\left./ \mathrm{s}\right)$ for a $65 \times 65 \mathrm{~nm}^{2}$ detection pixel, Eq. (1) predicts $B_{\min } \simeq$ $7.4 \mu \mathrm{T}$ for $t=1 \mathrm{~s}$. Experimentally, we observe a detection threshold that is within $15 \%$ of the photoelectron shotnoise limit [Fig. 1(e)]. The experimentally determined $B_{\text {min }}$ is calculated as the standard deviation of field values in $65 \times 65 \mathrm{~nm}^{2}$ pixels in magnetic images lacking visible features. Scaling of the experimental detection threshold to a $390 \times 390 \mathrm{~nm}^{2}$ pixel (approximately the diffractionlimited resolution), gives $B_{\min } \simeq 1.4 \mu \mathrm{T}$ for $t=1 \mathrm{~s}$. This detection threshold is a factor of six lower than that for $65 \times 65 \mathrm{~nm}^{2}$ pixels because $I_{0}$ increases by a factor of 36 [Eq. (1)].

This detection threshold is sufficient to image the microtesla fields from hemozoin nanocrystals. In our experimental geometry [Fig. 1(d)], hemozoin crystals are magnetized along the $x$ axis and the $B_{x}$ component of the magnetic field is detected by diamond magnetic microscopy. In the point-dipole approximation [35], which is valid for crystals with dimensions smaller than the 390-nm spatial resolution of our microscope, the magnetic field produced from a single crystal (volume $V$ ) is

$$
B_{x}(x, y, z)=\frac{\chi V B_{0}}{4 \pi} \frac{2 x^{2}-y^{2}-z^{2}}{\left(x^{2}+y^{2}+z^{2}\right)^{5 / 2}},
$$

where $B_{0}$ is the applied magnetic field and $\chi$ is the volume magnetic susceptibility. $\chi$ depends on the crystal orientation and purity, and values of $(3.2-4.6) \times 10^{-4}$ have been reported in the literature $[10,18]$. For conservative values $\chi=3.2 \times 10^{-4}, V=100 \times 100 \times 100 \mathrm{~nm}^{3}$, and NV sensing depth $z=200 \mathrm{~nm}$, the field produced by a hemozoin nanocrystal in a $B_{0}=350 \mathrm{mT}$ applied field has a minimum of $B_{x}=-1.1 \mu \mathrm{T}$ at $x=y=0$ and maxima of $B_{x}=0.2 \mu \mathrm{T}$ at $y=0, x= \pm 245 \mathrm{~nm}$.

To more accurately describe the instrument response, magnetostatic modeling is used to calculate the $B_{x}(x, y, z)$ 

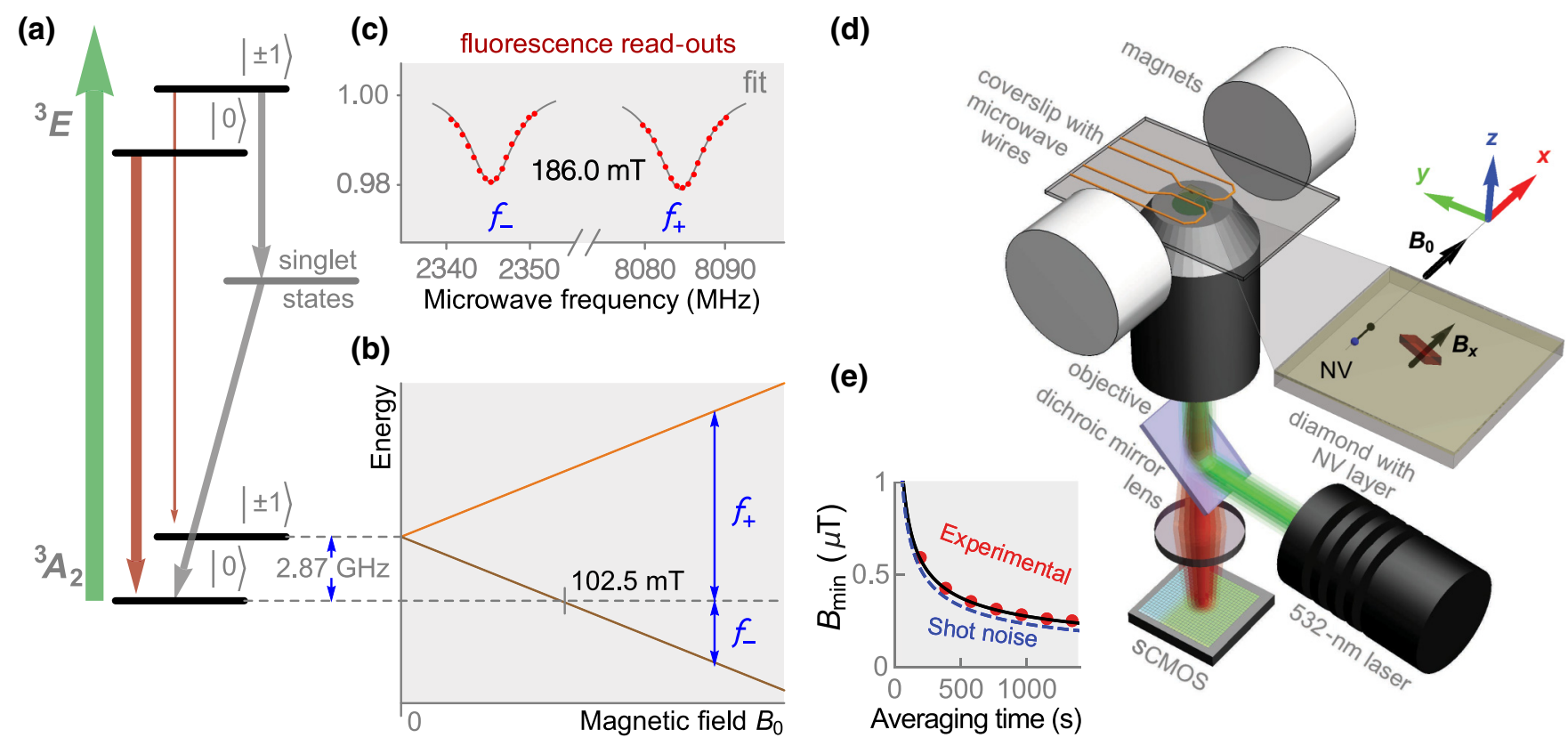

FIG. 1. Diamond magnetic microscopy. (a) NV energy-level diagram depicting magnetic sublevels $(|0\rangle,| \pm 1\rangle)$, optical excitation (green arrow), fluorescence (red arrows), and nonradiative (gray arrows) pathways. Gray arrows show spin-selective intersystem crossing leading to polarization into the $|0\rangle$ ground-state sublevel. (b) Energy splitting of the ground-state sublevels in an external magnetic field applied along the NV axis. Blue arrows indicate the $f_{ \pm}$microwave transitions. (c) Example of an ODMR spectrum. From the separation between peaks, the projection of the local magnetic field along the NV axis is inferred. (d) Epifluorescence ODMR microscope used for magnetic imaging. Dry hemozoin crystals are placed on top of a diamond substrate with an $\sim 0.2-\mu \mathrm{m}$ top layer doped with NV centers. (e) Experimental and photoelectron-shot-noise-limited detection threshold for $65 \times 65 \mathrm{~nm}^{2}$ detection pixels versus averaging time. The experimental data are fit to the function $B_{\min }=\alpha / \sqrt{t}$, with $\alpha=8.4 \pm 0.1 \mu \mathrm{T} \mathrm{s}{ }^{1 / 2}$.

field produced by elongated crystals, with dimensions taken from scanning-electron-microscopy (SEM) images. We then integrate $B_{x}$ over the NV vertical distribution, which is assumed to be uniform from 20 to $220 \mathrm{~nm}$ below the diamond surface (Appendix B). Finally, we account for the effect of optical diffraction and image drift by convolution with a two-dimensional Gaussian kernel ("blur") with 540-nm FWHM. A discussion of model parameters is given in Appendix F.

\section{EXPERIMENTAL METHODS}

Figure 1(d) depicts the epifluorescence ODMR microscope used for diamond magnetic microscopy. The diamond substrate is a [110]-polished, $2 \times 2 \times 0.08 \mathrm{~mm}^{3}$ type-Ib-diamond substrate grown by high-pressure, hightemperature synthesis. ${ }^{4} \mathrm{He}^{+}$ions were implanted into the substrate [31] at three different energies $(5,15$, and 33 $\mathrm{keV}$ ) to produce a roughly uniform distribution of vacancies in an $\sim 200$-nm near-surface layer (see Appendix B). After implantation, the diamond was annealed in a vacuum furnace [36] at $800^{\circ} \mathrm{C}(4 \mathrm{~h})$ and $1100^{\circ} \mathrm{C}(2 \mathrm{~h})$ to produce a near-surface layer of $\mathrm{NV}$ centers with a density of $\sim 10 \mathrm{ppm}$. Microwaves are delivered by copper loops printed on a contacting glass coverslip. A magnetic field,
$\vec{B}_{0}$, produced by a pair of permanent magnets, points along one of the in-plane NV axes. A linearly polarized 532-nm laser beam $(0.2 \mathrm{~W})$ excites the $\mathrm{NV}$ centers over an area of $\sim 40 \times 40 \mu \mathrm{m}^{2}$, and their fluorescence is imaged onto a scientific CMOS (sCMOS) camera. A detailed description of the apparatus is given in Appendix B.

Magnetic field maps are obtained by performing ODMR imaging of the near-surface NV layer (see Appendix D). Fluorescence images $(600$ pixels $\times 600$ pixels, $39 \times$ $39 \mu \mathrm{m}^{2}$ field of view, 3-ms exposure time) of the NV layer are recorded at 16 different microwave frequencies around each of the NV ODMR resonances (32 frequencies in total). The sequence is repeated, and the image set is integrated for several minutes to increase the signal-tonoise ratio. The fluorescence-intensity-versus-microwavefrequency data for each pixel are fit by Lorentzian functions to determine the ODMR central frequencies, $f_{ \pm}$. The magnetic field projection along the NV axis is then calculated as $B_{\|} \equiv B_{0}+B_{x}=\left(f_{+}-f_{-}\right) /\left(2 \gamma_{\mathrm{NV}}\right)$. Determining $B_{\|}$in this way eliminates common-mode shifts in $f_{ \pm}$ due to temperature-dependent changes in zero-field splitting $D$ [37] and variations in longitudinal strain $[33,38]$. The external field, $B_{0}$, is subtracted from the image, revealing a map of the stray magnetic fields, $B_{x}$, produced by the magnetized nanocrystals. 

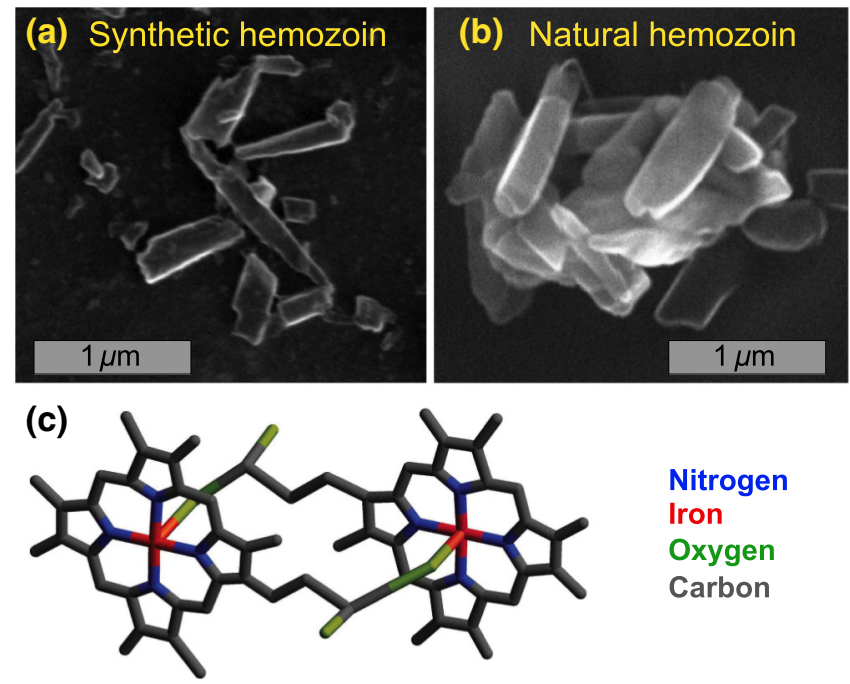

Nitrogen

Iron

Oxygen

Carbon

FIG. 2. Hemozoin nanocrystals. (a) SEM image of synthetic hemozoin nanocrystals on a diamond substrate. (b) SEM image of natural hemozoin nanocrystals extracted from human red blood cells cocultured with Plasmodium falciparum, on a diamond substrate. (c) Molecular structure of a hematin dimer, which can form hemozoin crystals when joined together by hydrogen bonds. The molecular structure is expected to be the same for natural and synthetic hemozoin crystals.

Figure 2 shows SEM images of the synthetic and natural hemozoin nanocrystals studied here. The synthetically produced hemozoin crystals (tlrl-hz, InvivoGen) have an elongated shape and varied dimensions $(50-1500 \mathrm{~nm})$. The natural hemozoin nanocrystals, extracted from human red blood cells cocultured with Plasmodium falciparum [24], have similar elongated shapes with slightly larger average dimensions. For diamond magnetic microscopy, hemozoin samples are diluted in water and drop-cast on the diamond surface to yield a relatively nonaggregated surface density with approximately 0.04 nanocrystals $/ \mu \mathrm{m}^{2}$.

\section{RESULTS}

Figure 3(a) shows a bright-field transmission image of natural hemozoin crystals dispersed on the diamond sensor's surface. Most of the bright features in the image come from host-cell residue; they do not appear in magnetic images. Results from another region without residue are reported in Appendix 3.

Figure 3(b) displays a modified SEM image of the same region as in Fig. 3(a). We use an image-segmentation procedure (Appendix C) to more clearly visualize the hemozoin crystals. Figure 3(c) shows the corresponding magnetic field map obtained by diamond magnetic microscopy. Of a total of 120 features identified as potential hemozoin nanocrystals in the SEM image, 82 exhibit magnetic features resolved by our technique. Surprisingly, the two magnetic features with the largest magnitude correspond to crystals with dimensions of $\sim 200 \mathrm{~nm}$ or less in the SEM image. Such anomalously strong features are observed consistently, but infrequently (less than $5 \%$ of all magnetic features), in both natural and synthetic hemozoin samples. We tentatively attribute them to superparamagnetism [39], for reasons discussed below.

Five example crystals are labeled $\mathrm{n} 1-\mathrm{n} 5$ in the images in Figs. 3(b) and 3(c), including one of the aforementioned strongly magnetized nanocrystals (n5). Figure 4(a) shows SEM images of each example crystal; $n 1-n 4$ have a typical size and shape for these crystals, whereas $\mathrm{n} 5$ is barely visible, with dimensions of $<200 \mathrm{~nm}$. Figure 4(b) shows the corresponding diamond-magnetic-microscopy images taken at $B_{0}=186 \mathrm{mT}$. Each crystal exhibits a different field pattern characteristic of its unique size, shape, and orientation.

Figure 4(c) shows the expected magnetic field patterns of each nanocrystal, calculated with the procedure described in Sec. II. Each nanocrystal is modeled as a three-dimensional (3D) ellipsoid with uniform susceptibility and dimensions inferred from the corresponding
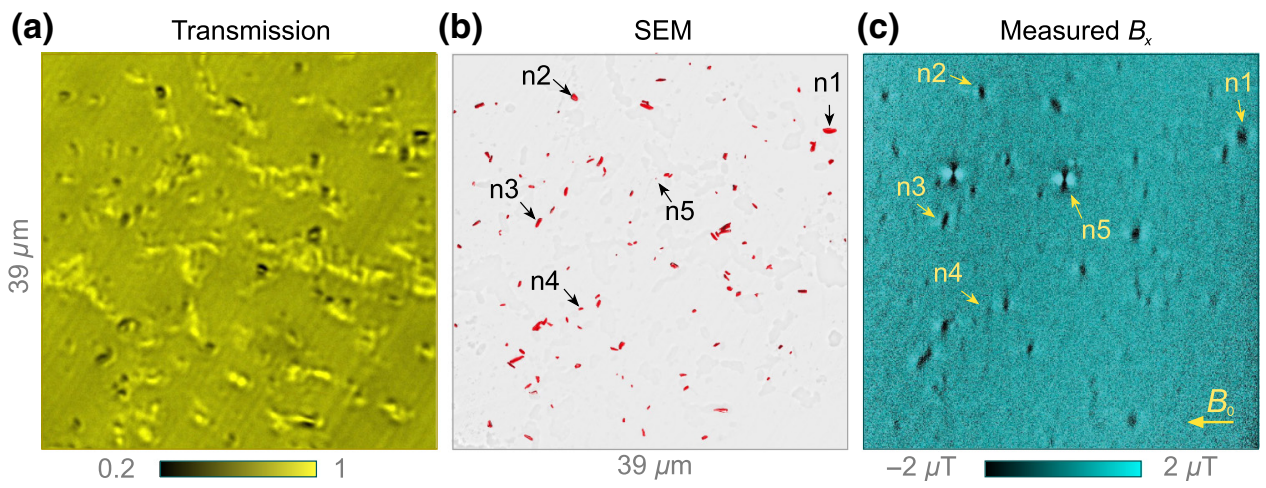

FIG. 3. Natural hemozoin. (a) Bright-field transmission image of natural hemozoin crystals dispersed on a diamond substrate. The scale bar is the relative transmission. (b) SEM image of the same nanocrystals. To enhance hemozoin visibility, the SEM image is modified by a segmentation procedure, depicted in Fig. 7. (c) Diamond-magnetic-microscopy image for applied field $B_{0}=350 \mathrm{mT}$. Nanocrystals labeled n1-n5 are studied in detail in Figs. 4(a)-4(e). 


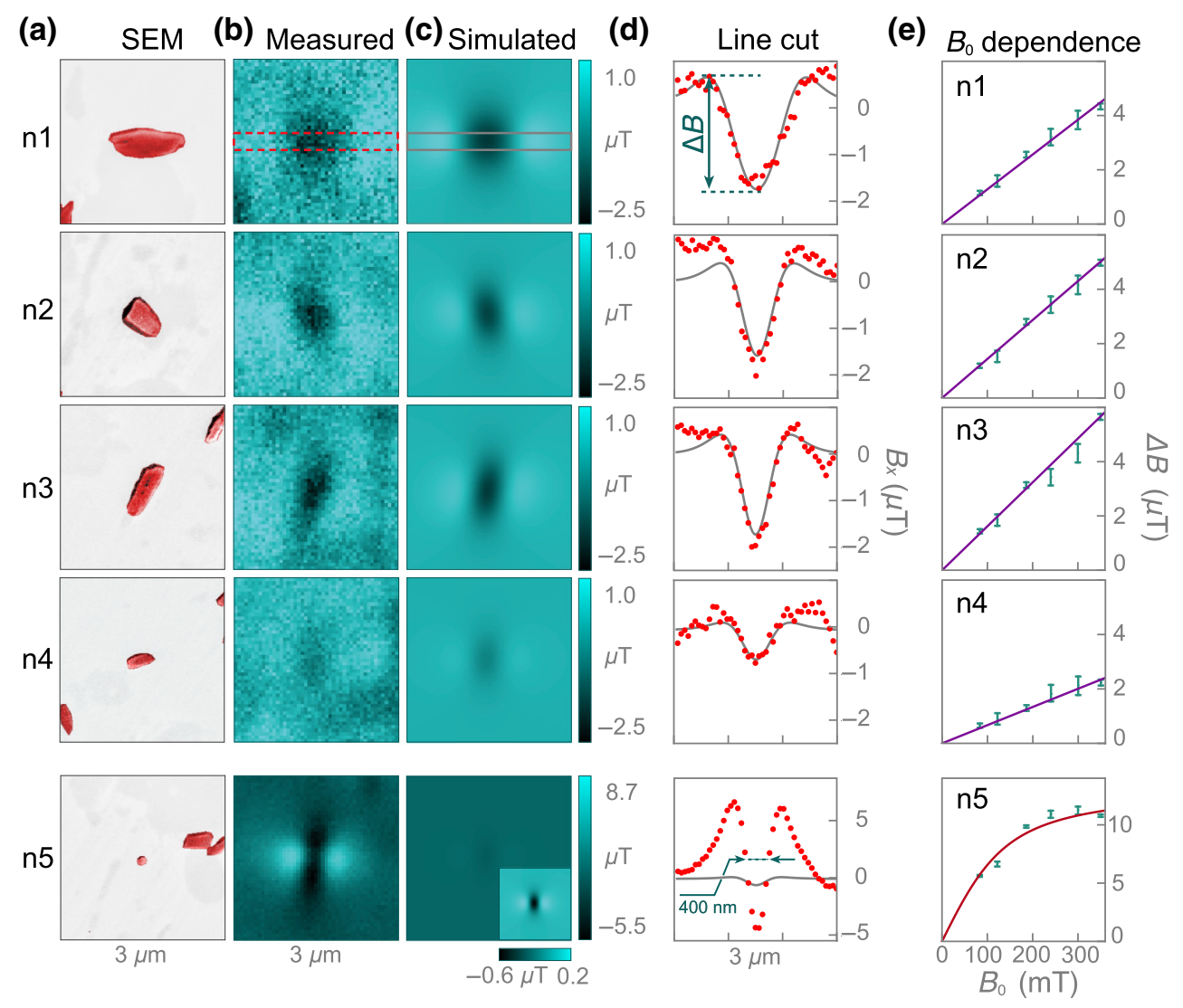

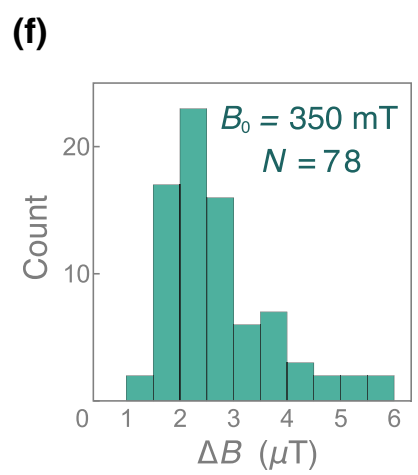

(g)

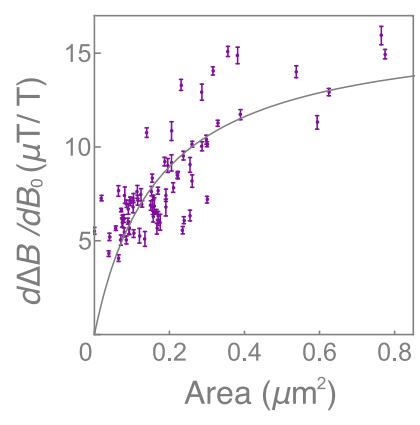

FIG. 4. Magnetic imaging of individual natural hemozoin nanocrystals. (a) SEM images of hemozoin nanocrystals n1-n5, labeled in Fig. 3. (b) Corresponding diamond-magnetic-microscopy images $\left(B_{0}=186 \mathrm{mT}\right)$ for each crystal. (c) Simulated magnetic images $\left(\chi=3.4 \times 10^{-4}, B_{0}=186 \mathrm{mT}\right)$ obtained with nanocrystal dimensions inferred from (a). Only the crystal at the center of each SEM image is included in the model. (d) Line cuts of each nanocrystal (red, measured; gray, simulated), from which the field-pattern amplitude $\Delta B$ is inferred. A minimum feature width of $\sim 400-\mathrm{nm}$ FWHM is observed for n5, close to the optical diffraction-limited resolution of our microscope. (e) $\Delta B\left(B_{0}\right)$ for each crystal. Solid lines are weighted fits to a line (n1-n4) or Langevin function (n5). All fits are constrained to intercept the origin, $\Delta B(0)=0$ (zero-coercivity assumption). (f) Histogram of $\Delta B\left(B_{0}=350 \mathrm{mT}\right)$ of the 78 crystals exhibiting linear paramagnetic behavior (including n1-n4). The four crystals exhibiting superparamagnetic behavior (including n5) are excluded from the analysis. (g) Fitted slopes, $d \Delta B / d B_{0}$, as a function of crystal area, $A$, as determined from SEM images. The data are fitted with an empirical saturation function, $d \Delta B / d B_{0}=S_{\max } /\left(1+A_{\text {sat }} / A\right)$, with $S_{\max }=16.5 \pm 1.4 \mu \mathrm{T} / \mathrm{T}$ and $A_{\text {sat }}=0.17 \pm 0.03 \mu \mathrm{m}^{2}$.

SEM images. The height of the crystals is assumed to be $200 \mathrm{~nm}$. For $\mathrm{n} 1-\mathrm{n} 4$, the model produces field patterns similar to those observed experimentally. The pattern amplitude is best described with a volume magnetization $M=50 \mathrm{~A} / \mathrm{m}$, which corresponds to a volume susceptibility $\chi=\mu_{0} M / B_{0}=3.4 \times 10^{-4}$, where $\mu_{0}=4 \pi \times$ $10^{-7} \mathrm{~m} \mathrm{~T} / \mathrm{A}$ is the vacuum permeability. This value of $\chi$ is well within the range of literature values for hemozoin $[10,18]$ and is in good agreement for most crystals, despite a wide variation in their magnetic patterns. This demonstrates that crystal size, shape, and orientation are the primary factors in determining the magnetic pattern behavior. Factors contributing to $\sim 25 \%$ or less uncertainty in $\chi$ are discussed in Appendix F.

For crystal n5, however, the model does a poor job of describing the magnetic behavior. It predicts a field-pattern amplitude more than one order of magnitude lower than the observed value, suggesting $\mathrm{n} 5$ is not paramagnetic hemozoin. It is difficult to estimate this particle's magnetization, since it may arise from a small inclusion or an adjacent particle not resolved in the SEM image.

Line cuts of the magnetic field patterns for $n 1-n 5$ are shown in Fig. 4(d). The line cuts are obtained by averaging $B_{x}$ values over six rows $(390 \mathrm{~nm})$ in a band along the magnetic feature, as indicated in Figs. 4(b) and 4(c). The line cuts are used to calculate the magnetic pattern amplitude $\Delta B$, determined from the difference in extreme $B_{x}$ values in the line cuts. The uncertainty in $\Delta B$ is approximately $\pm 0.2 \mu \mathrm{T}$, on the basis of the scatter in the $B_{x}$ values in the line cuts in a magnetic-feature-free region. The amplitudes for $\mathrm{n} 1-\mathrm{n} 4$ differ due to size and shape, but all fall in the range $\Delta B=1.3-2.9 \mu \mathrm{T}$. However, the 
amplitude for $\mathrm{n} 5$ is much larger, $\Delta B=11.0 \pm 0.2 \mu \mathrm{T}$. The FWHM of this feature is $\sim 400 \mathrm{~nm}$, close to our microscope's diffraction-limited spatial resolution, providing further evidence that it comes from a pointlike particle.

Magnetic images of all nanocrystals shown in Fig. 3 were obtained for six values of the external field: $B_{0}=83$, $122,186,240,300$, and $350 \mathrm{mT}$. From these images, we identify 82 individual magnetized crystals and calculate $\Delta B$ as a function of $B_{0}$ for each of them. The $\triangle B\left(B_{0}\right)$ curves for all 82 crystals are provided in Fig. 13. Figure 4(e) shows the curves of nanocrystals $n 1-n 5$. A linear dependence is found for $\mathrm{n} 1-\mathrm{n} 4$ and is characteristic of a paramagnetic response. In total, 78 of 82 natural hemozoin crystals show a similar linear behavior, with a slope in the range $d \Delta B / d B_{0}=4-16 \mu \mathrm{T} / \mathrm{T}$.

The remaining four nanoparticles from the natural hemozoin sample exhibit a saturation behavior, as seen for n5. These curves are fit with a Langevin function of the form $\Delta B\left(B_{0}\right)=a\left[\operatorname{coth}\left(b B_{0}\right)-1 /\left(b B_{0}\right)\right]$, where $a$ and $b$ are fit parameters. For n5, $a=13.3 \pm 0.7 \mu \mathrm{T}$ and $b=0.017 \pm 0.002 \mathrm{mT}^{-1}$. This model is commonly used to describe superparamagnetic nanoparticles in the limit that the thermal energy exceeds the magnetic anisotropy energy [40]. The large magnetization and saturation behavior observed in these small nanoparticles is consistent with recent reports of superparamagnetism in hemozoin samples $[39,41]$. However, only a small fraction $(\sim 5 \%$ or less) of the nanoparticles exhibit such behavior. This may explain why superparamagnetic behavior was not observed in previous ensemble studies with unrefined samples $[18,22,25]$. The atomic structure of these outlier nanoparticles remains a topic for future work.

A histogram of $\Delta B$ for all 78 paramagnetic nanocrystals at $B_{0}=350 \mathrm{mT}$ is shown in Fig. 4(f). The distribution is characterized by a mean amplitude of $2.9 \mu \mathrm{T}$, a median of $2.6 \mu \mathrm{T}$, and a standard deviation of $1.6 \mu \mathrm{T}$. Figure 4(f) plots the best-fit slopes, $d \Delta B / d B_{0}$, as a function of crystal area, as determined from SEM images. The slopes increase in a roughly monotonic fashion before saturating when the crystal dimensions exceed the spatial resolution of the microscope. This behavior is expected for crystals with uniform susceptibility. For crystal volumes much smaller than the sensing voxel, $V \ll V_{\text {sense }} \approx 0.4 \times$ $0.4 \times 0.2 \mu \mathrm{m}^{3}$, Eq. (2) predicts $d \Delta B / d B_{0} \propto \chi V$, while for $V \gg V_{\text {sense }}$ we expect $d \Delta B / d B_{0} \propto \chi$ independent of crystal dimensions.

We have also measured the magnetic properties of synthetic hemozoin crystals manufactured by InvivoGen. These nanocrystals are commonly used as a model for natural hemozoin, owing to their ease of procurement and nearly identical crystal morphology and chemical structure $[42,43]$. Previous studies reported similarities in the ensemble magnetic properties of synthetic and Plasmodium falciparum-extracted hemozoin [44]; however, here we compare the distribution of their magnetic properties at the single-nanocrystal level.

Figure 5(a) shows a confocal reflection image of dried synthetic hemozoin crystals dispersed on the diamond substrate. Of a total of 46 dark features identified as potentially being hemozoin, 41 produce magnetic features in the diamond-magnetic-microscopy images [Fig. 5(b)]. As with natural hemozoin, $\Delta B\left(B_{0}\right)$ curves are generated by monitoring these features in magnetic images taken at six different applied fields. The $\Delta B\left(B_{0}\right)$ curves for all 41 nanocrystals are displayed in Fig. 14. One nanocrystal exhibits a Langevin saturation, suggesting superparamagnetic behavior, while the remaining 40 crystals exhibit a linear dependence. Figure 5(c) shows the $\Delta B\left(B_{0}\right)$ curves for three example nanocrystals exhibiting linear behavior, labeled s1-s3 in Fig. 5(b). Magnetostatic modeling [Fig. 10(d)] of these three nanocrystals shows good agreement with the experimental images with $\chi=3.4 \times 10^{-4}$. This is the same value as found for natural hemozoin [Figs. 4(c) and 4(d)], indicating the natural and synthetic crystals have similar magnetic properties.

Figure 5(d) shows histograms of the fitted slopes, $d \Delta B / d B_{0}$, for all 40 paramagnetic synthetic crystals and all 78 paramagnetic natural crystals. The synthetic nanocrystals have a slightly smaller slope (mean 5.5,
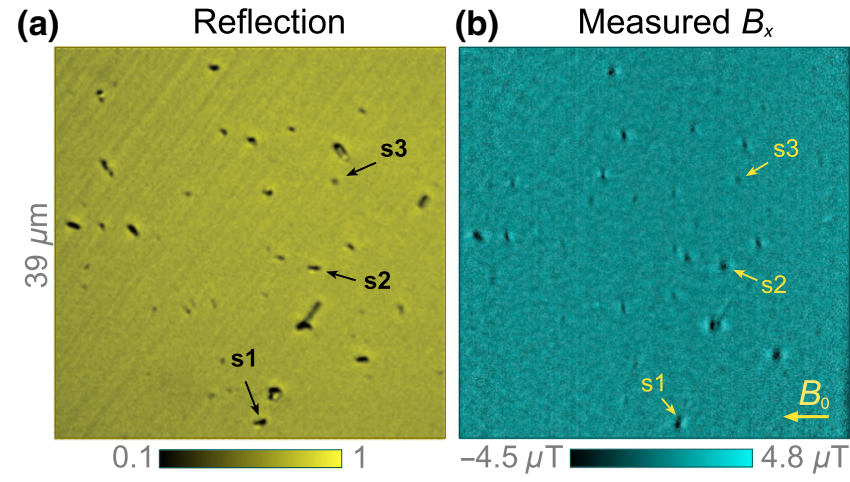

(c)

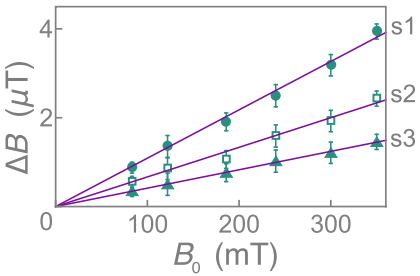

(d)

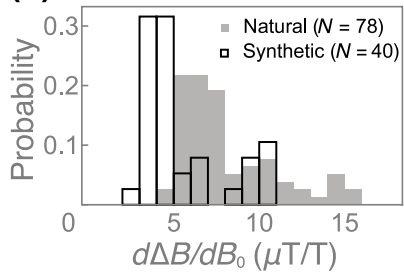

FIG. 5. Synthetic hemozoin. (a) Confocal reflection image (excitation at $405 \mathrm{~nm}$ ) of synthetic hemozoin nanocrystals on a diamond substrate. In total, 46 dark features are identified as likely hemozoin nanocrystals. (b) Corresponding diamondmagnetic-microscopy image at $B_{0}=350 \mathrm{mT}$. 41 of 46 possible crystals exhibit an observable magnetic feature. (c) $\Delta B\left(B_{0}\right)$ curves for three synthetic hemozoin crystals labeled in (b). Solid lines are weighted linear fits. (d) Histograms of $d \Delta B / d B_{0}$ for natural and synthetic hemozoin crystals. 
median $4.3 \mu \mathrm{T} / \mathrm{T}$ ) than natural hemozoin (mean 8.1, median $7.2 \mu \mathrm{T} / \mathrm{T}$ ), while the standard deviation is simi$\operatorname{lar}(6.2$ and $8.0 \mu \mathrm{T} / \mathrm{T}$, respectively). This is likely due to a small difference in their size distributions (see Appendix C and Fig. 8).

\section{OUTLOOK AND CONCLUSION}

Our results demonstrate the capability of diamond magnetic microscopy to simultaneously measure the magnetic properties of numerous individual biocrystals. Whereas bulk measurements yield ensemble-average properties, diamond magnetic microscopy can measure the distribution of nanocrystal susceptibilities, extract information about each crystal's dimensions and orientation, and differentiate paramagnetic hemozoin from superparamagnetic nanoparticles. This brings up the intriguing possibility of using this platform to monitor the formation dynamics of individual hemozoin nanocrystals in living cells without the use of extrinsic contrast agents.

To assess the feasibility of performing magnetic imaging of hemozoin inside cells, we estimate a typical hemozoin crystal volume is $V=0.04 \mu \mathrm{m}^{3}$, on the basis of the median area found from SEM images $\left(0.2 \mu \mathrm{m}^{2}\right)$ and an assumed thickness of $0.2 \mu \mathrm{m}$. We assume the hemozoin crystal forms in a digestive vacuole within a Plasmodium-infected red blood cell (typical thickness $2 \mu \mathrm{m}$ [13]) such that it is located $2 \mu \mathrm{m}$ from the diamond surface. Such a crystal with susceptibility $\chi=3.4 \times 10^{-4}$ under an applied field $B_{0}=350 \mathrm{mT}$ would produce a magnetic pattern amplitude at the diamond surface $(z=$ $2 \mu \mathrm{m}$ ) of $\Delta B=0.057 \mu \mathrm{T}$ [Eq. (2)]. This field shift would be detectable with a signal-to-noise ratio of one after $\sim 10 \mathrm{~min}$ of averaging using the present diamond sensor with $390 \times 390 \mathrm{~nm}^{2}$ detection pixels (see Sec. II). However, the 200-nm NV layer currently used is optimized for negligible diamond-hemozoin separation. If the separation is $\sim 2 \mu \mathrm{m}$, an NV layer of $1 \mu \mathrm{m}$ would be more optimal, leading to a signal-to-noise ratio of one after approximately $2 \mathrm{~min}$. If a higher magnetic field is used (already realized for nanoscale NV magnetometry $[45,46])$, a signal-to-noise ratio of one should be obtained for $B_{0}=3 \mathrm{~T}$ after $\sim 1.6 \mathrm{~s}$ of signal averaging.

This sensitivity would be sufficient to monitor the formation dynamics [47] of individual hemozoin nanocrystals on minute-to-hour timescales. It could enable monitoring of crystal position and orientation throughout the parasite life cycle under different conditions, including the influence of antimalarial drugs. However, the movement of red blood cells would need to be constrained such that crystals remain close to the diamond surface. This may be accomplished by promotion of cell adhesion [48] or sedimentation [49], confinement of cells within microstructures $[36,50]$, and/or increase of the viscosity of the surrounding medium [51-53]. If the platform is simplified and miniaturized, it may also find application as a labelfree malaria diagnostic tool with sensitivity rivaling the current staining or microscopy standard [18]. Our platform can also be used to study paramagnetic substances other than hemozoin. Figure 11 shows diamond-magneticmicroscopy images of the pharmacological agent hemin.

In summary, we use diamond magnetic microscopy to characterize the distribution of magnetic properties of synthetic and natural hemozoin samples at the individual nanocrystal level. More than $95 \%$ of the nanocrystals exhibit paramagnetic behavior, with magnetic field patterns well described by a magnetostatic model using a volume susceptibility $\chi=3.4 \times 10^{-4}$. Five of the 123 nanocrystals studied exhibit anomalously large magnetization that saturates at fields above approximately $0.1 \mathrm{~T}$, suggesting superparamagnetism. Further work is needed to determine the composition and structure of these outlier nanoparticles. With improvements in the experimental setup, diamond magnetic microscopy should be capable of imaging hemozoin formation dynamics in living cells, with implications for malaria diagnosis and drug development.

\section{ACKNOWLEDGMENTS}

The authors acknowledge valuable conversations with C. Belthangady, D. Budker, Y. Silani, and J. Damron, as well as sample characterization resources at the UNM Comprehensive Cancer Center Fluorescence Microscopy Shared Resource and the U.S. Department of Energy Center for Integrated Nanotechnologies. This work was funded by NIH (National Institute of General Medical Sciences) Grant No. 1R41GM130239-01, NIH (National Institute of Mental Health) Grant No. 1R41MH115884-01, NIH (National Institute of Biomedical Imaging and Bioengineering) Grant No. 1R01EB025703-01, and a Beckman Young Investigator award.

L.B., A.J., and V.M.A. are named as coinventors on a related U.S. patent application: 20180203080 A1. A.J. and L.B. are cofounders of ODMR Technologies and have financial interests in the company. The remaining authors declare no competing financial interests.

L.B., P.K., and (later) V.M.A. conceived the idea for this study in consultation with A.J.. P.K. performed initial calculations demonstrating feasibility. I.F., A.J., and V.M.A. designed the experimental plan with input from all authors. J. Smits, P.K., and I.F. wrote and implemented control and automation software. J. Seto extracted the natural hemozoin samples. I.F., A.L., and N.M. built the measurement apparatus, prepared samples, and collected data. I.F. analyzed the data and wrote the initial manuscript draft, under the supervision of V.M.A. All authors discussed the results and contributed to the writing of the manuscript. 


\section{APPENDIX A: HEMOZOIN}

Hemozoin crystals consist of dimers of $\alpha$-hematin molecules each containing an iron center. Figure 2 shows the molecular structure of a dimer, constructed with the Avogadro open-source molecular builder and visualization tool. The central iron of the first $\alpha$-hematin is bonded to the oxygen of the carboxylate side chain of the second $\alpha$-hematin. The dimers are joined by hydrogen bonds to form a triclinic crystal [47]. These crystals are alternatively called "hemozoin" or " $\beta$-hematin." Throughout this paper, we use the term "hemozoin" to refer to crystals produced both naturally (through biocrystallization inside living organisms) and synthetically.

The organisms that produce hemozoin crystals include the various Plasmodium species, the parasitic worm Schistosoma mansoni, the avian protozoan parasite Hemoproteus columbae, and the kissing bug Rhodnius prolixus [47]. In humans, hemozoin plays an important role in the pathological mechanism of the Plasmodium parasites responsible for malaria, a disease that has devastating effects in tropical and subtropical regions [10].

During their life cycle, malarial protozoans feed on host-cell hemoglobin by decomposing it inside digestive vacuoles into amino acids and toxic $\alpha$-hematin radicals [10-13]. The iron radicals are then bound, inside the same vacuoles, into inert hemozoin crystals and released into the host blood during erythrocyte disintegration.

The suppression (or alteration) of hemozoin formation inside Plasmodium parasites is considered a key action of widely used antimalarial drugs, such as quinine and chloroquine $[11,12]$. Quinine has been successfully used for more than 300 years to treat malaria by suppressing hemozoin crystallization [12]. Chloroquine has been widely used since the 20th century and is thought to invoke a similar response in hemozoin formation [11,12]. The regular use of these drugs led to the selection of resistant forms of Plasmodium parasites, which carry genes responsible for preventing the drugs from entering digestive vacuoles $[11,12]$.

The motivation for the present study was to establish diamond magnetic microscopy as an effective tool for fast, quantitative, noninvasive characterization of individual hemozoin nanocrystals. Translation of this technique to the study of hemozoin formation in living protozoans could be used to elucidate the interaction between hemozoin formation, the host immune system, and antimalarial drugs. This line of inquiry may even lead to the establishment of a bank of effective antimalarial drugs able to counteract the highly adaptive parasites.

\section{APPENDIX B: EXPERIMENTAL DETAILS}

The diamond substrate used in this work is a [110]polished, $2 \times 2 \times 0.08 \mathrm{~mm}^{3}$ type-Ib-diamond substrate grown by high-pressure, high-temperature synthesis. The diamond was acquired from Delaware Diamond Knives Inc. and had an initial nitrogen concentration of $\sim 100$ ppm. ${ }^{4} \mathrm{He}^{+}$ions were implanted in the substrate by CuttingEdge Ions LLC [31] at three different energies (5, 15 , and $33 \mathrm{keV}$ ) to produce a roughly uniform distribution of vacancies in an $\sim 200$-nm near-surface layer. Vacancy distribution depth profiles are estimated by Stopping and Range of Ions in Matter (SRIM) Monte Carlo simulation [Fig. 6(a)]. The diamond substrates are modeled as a pure carbon layer with density of $3.52 \mathrm{~g} / \mathrm{cm}^{3}$ and atom-displacement threshold energy of $37.5 \mathrm{eV}$. The lattice-damage threshold and surface-damage threshold are set to 7.35 and $7.5 \mathrm{eV}$, respectively. SRIM simulations do not take into account crystallographic effects such as ion channeling, and therefore could lead to underestimation of the vacancy-layer depth, but are sufficiently accurate for our purposes. Our SRIM simulations predict a modal depth of approximately $24 \mathrm{~nm}$ (the depth where the vacancy density is greatest) for $5-\mathrm{keV}^{4} \mathrm{He}^{+}$-ion implantation, approximately $72 \mathrm{~nm}$ for $15 \mathrm{keV}$, and approximately $130 \mathrm{~nm}$ for $33 \mathrm{keV}$. The diamond substrate was implanted with helium ion doses of $4 \times 10^{12}, 2 \times 10^{12}$, and $2 \times 10^{12} \mathrm{He} / \mathrm{cm}^{2}$ for implantation energies of 5, 15, and $33 \mathrm{keV}$, respectively. SRIM simulations [Fig. 6(a)], indicate this produces a vacancy density of approximately $50 \mathrm{ppm}$ with approximately $30 \%$ or less variation throughout the layer. After implantation, the diamond was annealed in a vacuum furnace at 800 and $1100^{\circ} \mathrm{C}$ [Fig. 6(b)]. This process resulted in an $\sim 200$-nm near-surface layer of NV centers with a

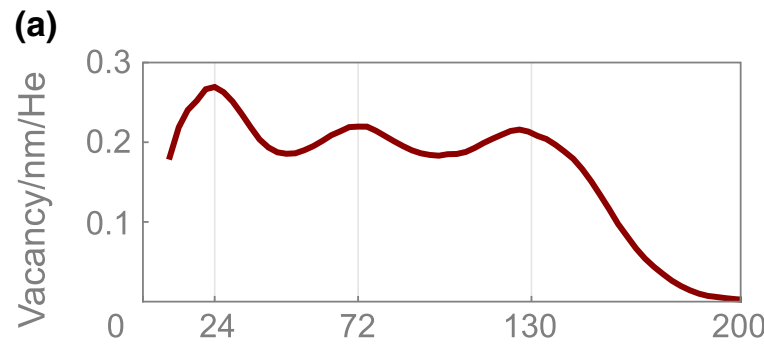

(b)

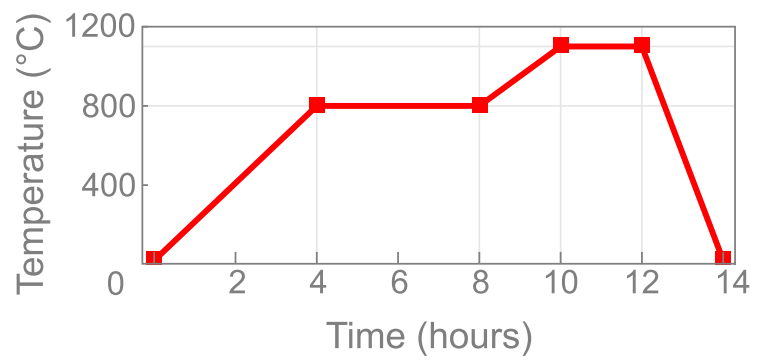

FIG. 6. NV-layer fabrication. (a) SRIM vacancy-depth profile for implantation conditions discussed in the text. (b) Timetemperature graph for the annealing procedure used in this study. 
density of approximately $10 \mathrm{ppm}$, as determined by the fluorescence intensity [30]. The largest source of uncertainty in the final NV-layer distribution is the extent of vacancy diffusion during annealing. Previous studies [54] predicted tens of nanometers of diffusion during annealing of type-Ib diamonds grown by high-pressure, high-temperature synthesis under similar conditions. We thus expect a slight broadening of the vacancy distribution in Fig. 6(a) to approximately $200 \mathrm{~nm}$ after annealing.

To magnetize nanocrystals, we use the static magnetic field created by a pair of permanent magnets [Fig. 1(d)]. The magnetic field is aligned to the [110]-polished diamond surface such that $\vec{B}_{0}$ is parallel to one of the two possible in-plane NV symmetry axes. Microwave fields are delivered by two copper loops (each addressing a different spin transition) printed on a glass coverslip. The loops are positioned with respect to the diamond to maximize the microwave-field components orthogonal to the NV axis.
The microwaves are generated by a Stanford Research Systems SG384 frequency generator with two outputs for the first and second harmonics. The frequency of the generator is swept by sending a ramp function to the generator's ana$\log$ frequency-modulation input. For $B_{0}>186 \mathrm{mT}$, additional frequency doublers (Mini-Circuits ZX90-2-24-S+ and ZX90-2-36-S+) are used on both harmonic outputs to access higher microwave frequencies. The microwaves for each transition are amplified separately by Mini-Circuits amplifiers: ZHL-16W-43-S+ (for microwave frequencies below $4 \mathrm{GHz}$ ), ZVE-3W-83+ (for 4-8 GHz), and ZVE$3 \mathrm{~W}-183+$ (for more than $8 \mathrm{GHz}$ ). The output of the amplifiers is subsequently routed to the coverslip-printed loops.

To produce NV fluorescence, $\sim 0.2 \mathrm{~W}$ of $532-\mathrm{nm}$ light from a Lighthouse Photonics Sprout-G-10W laser is used in a homebuilt inverted epifluorescence microscope [Fig. 1(d)]. We use an oil-immersion objective with

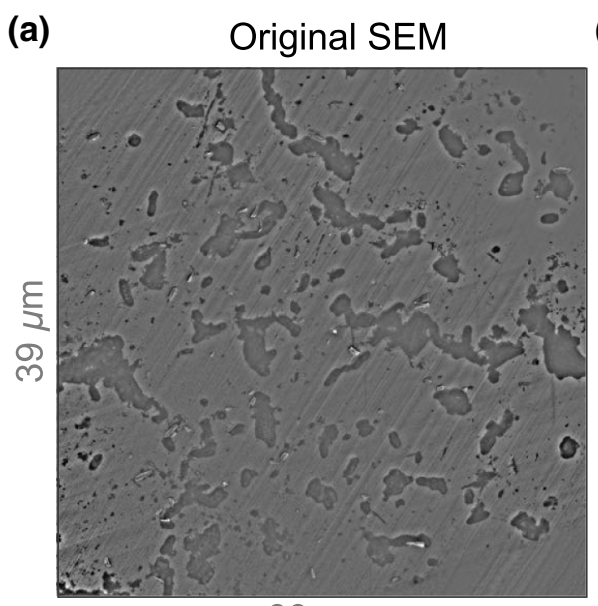

(d)

\section{$39 \mu \mathrm{m}$}

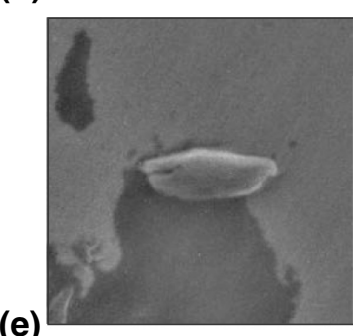

(e)
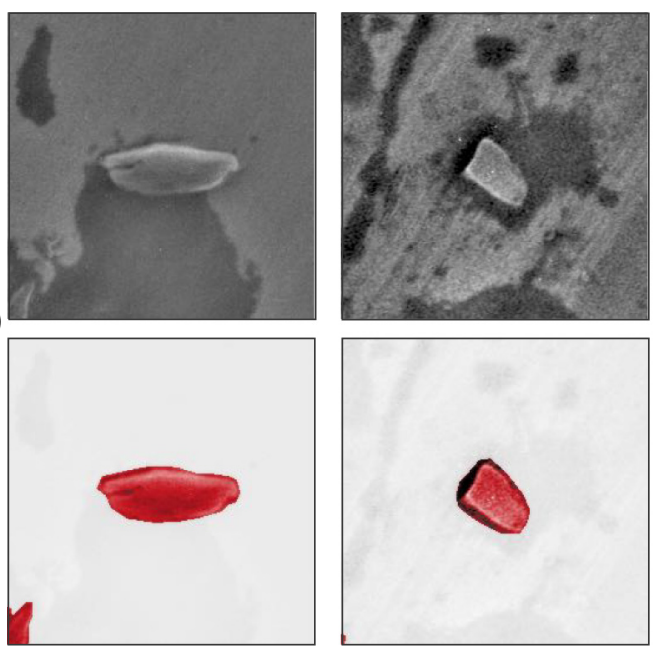

$\mathrm{n} 1$

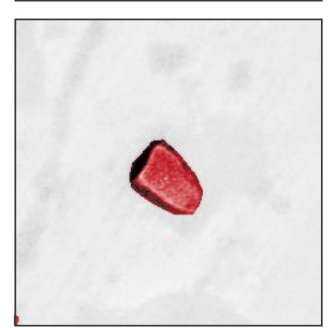

$\mathrm{n} 2$ (b) Mask

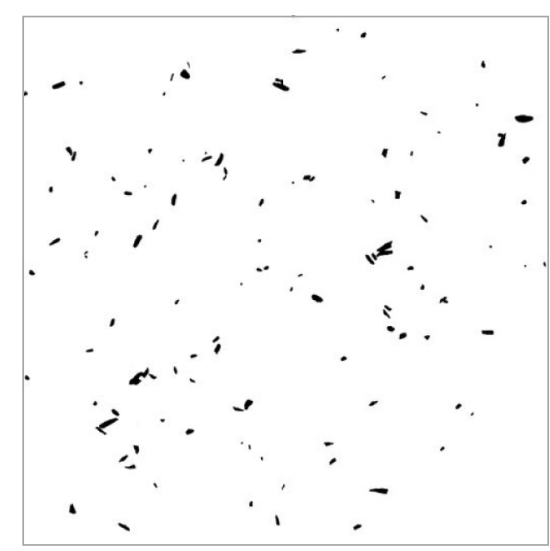

$39 \mu \mathrm{m}$ (c) Modified SEM

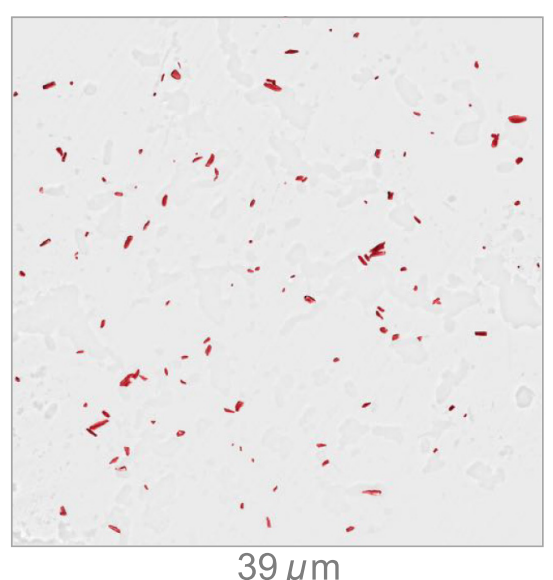

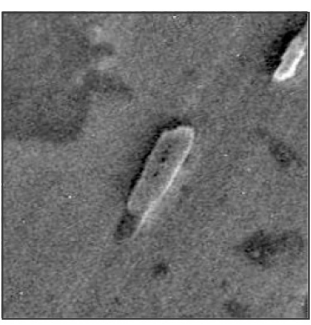
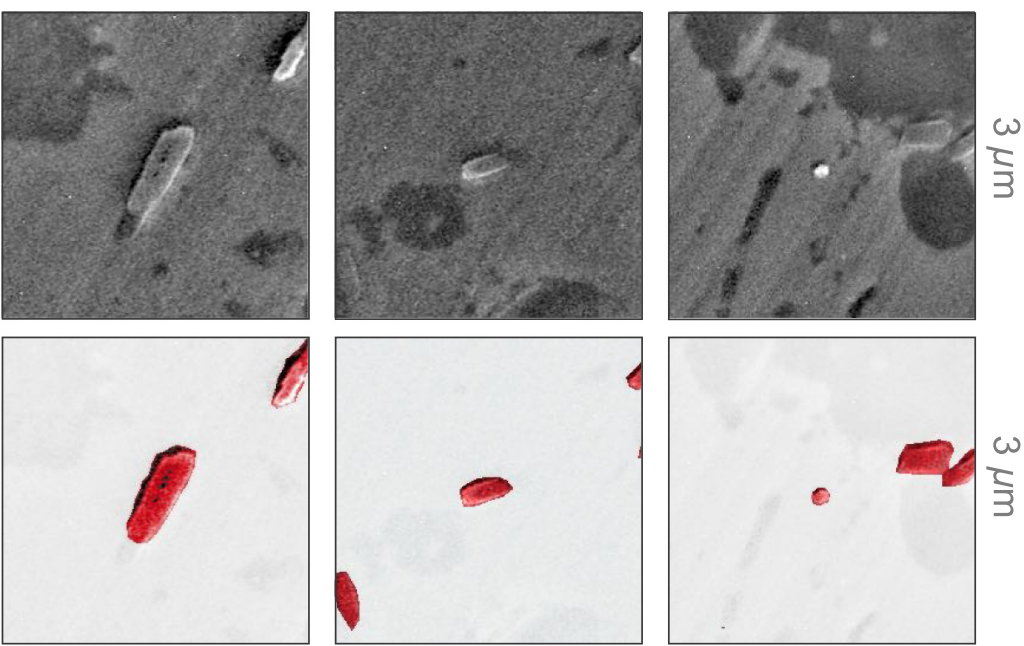

n3

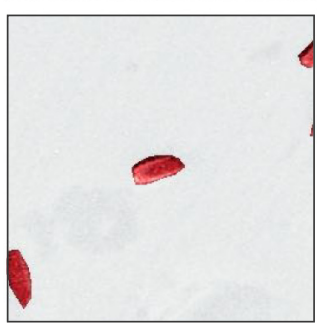

n4

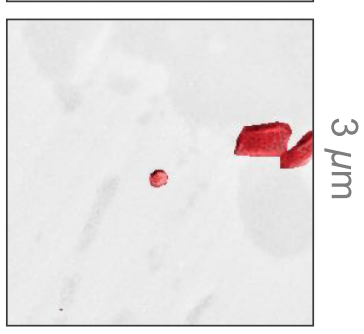

n5

FIG. 7. SEM processing. (a) Unprocessed SEM micrograph of the natural hemozoin sample. (b) A mask used to highlight and colorize hemozoin crystals and to attenuate their background. (c) Modified SEM image as shown in Fig. 3(b). (d) Unprocessed and (e) modified SEM images of hemozoin crystals n1-n5 addressed in Figs. 4(a)-4(e). 
a numerical aperture of 1.3, but an air gap between the coverslip and diamond reduces the diffraction-limited spatial resolution to $\sim 390 \mathrm{~nm}$. The laser light is focused to the lateral periphery of the objective's back focal plane to produce an illumination region of $\sim 40 \times 40 \mu \mathrm{m}^{2}$ on the diamond's top surface (the surface in contact with the dry hemozoin). By excitation in this way, the laser beam enters the bottom of the transparent diamond substrate at a steep angle, leading to substantial internal reflection from the top diamond-air interface. This is helpful in maximizing NV excitation intensity, while minimizing the intensity incident on hemozoin samples. We find no evidence of photodamage on hemozoin samples even after studying them under intense, continuous illumination for several days. For sensitive samples, a light-shield layer, $\sim 50 \mathrm{~nm}$ thick, can be used to block all light from interacting with the sample [55]. For example, coating the face of the diamond that interfaces with the sample with a metal layer (e.g., $\mathrm{Ag}$ or $\mathrm{Al}$ ) of $\sim 25 \mathrm{~nm}$ would result in reflection of all light, particularly when combined with excitation at an angle exceeding the total-internal-reflection angle. Adding an additional dielectric layer of $\sim 25 \mathrm{~nm}$ on top of the metal (e.g., $\mathrm{SiN}, \mathrm{SiO}_{2}$, or $\mathrm{Al}_{2} \mathrm{O}_{3}$ ) would further prevent any evanescent optical field from interacting with the sample even if cells are adhered to the top of the sensor.

The microscope is operated on a standard vibrationisolation optical table in a climate-controlled laboratory environment. The fluorescence is spectrally filtered by a dichroic mirror and a Semrock BrightLine 731/137 $\mathrm{nm}$ band-pass filter, focused by a $200-\mathrm{mm}$-focal-length achromatic lens (Thorlabs ITL200), and detected by a Hamamatsu Orca v2 sCMOS sensor. By alternation of a flip mirror, the fluorescence could also be detected by a Thorlabs APD410A avalanche photodiode connected to a Yokogawa DL9040 oscilloscope for faster tuning and alignment. The same microscope configuration is used to obtain bright-field transmission images by collecting white light transmitted through the analyte and diamond.

A LabVIEW program is used to control the experiment. A software command triggers the camera to initiate a burst of sixteen 3-ms frames in rolling-shutter mode. Just before the first frame, the camera provides a precisely timed transistor-transistor logic (TTL) pulse that is used to trigger a sequence generated by a National Instruments multifunction data-acquisition module. The data-acquisition module provides an analog ramp function that is used to sweep the microwave-generator frequency via its analog frequencymodulation input. Sixteen frames are acquired for each microwave sweep. After each sweep, the microwave generator is reconfigured via a general-purpose-interface-bus command to alternate between first and second harmonics to alternately address $f_{+}$or $f_{-}$transitions. Camera frame acquisition and real-time image processing are synchronized via the LabVIEW interface. The image-processing procedure is described in detail in Appendix D.

\section{APPENDIX C: HEMOZOIN PREPARATION AND CHARACTERIZATION}

Synthetic hemozoin crystals were purchased from InvivoGen $^{\mathrm{TM}}$ (tlrl-hz) and natural hemozoin crystals were obtained from human red blood cells co-cultured with Plasmodium falciparum parasites. The hemozoin samples were centrifuged several times (5 min at $6000 \mathrm{rpm}$ ) in deionized water to wash out salts and cell residues. They were subsequently sonicated to minimize aggregation, and finally homogeneously dispersed on the diamond surface by dropcasting the hemozoin suspension and wicking away the water until it had dried.

The shape, size, and orientation of the hemozoin crystals were investigated by SEM (UNM Center for High Technology Materials and DOE Center for Integrated Nanotechnologies) and confocal reflectance microscopy (UNM Comprehensive Cancer Center Fluorescence Microscopy Shared Resource). An SEM image for the synthetic hemozoin sample reported in the main text was not obtained due to the sample's early destruction. However confocal reflectance images $(405 \mathrm{~nm}$ excitation) were obtained, which offer a spatial resolution of $\sim 225 \mathrm{~nm}$.

Figure 7(a) shows the raw SEM image of the natural hemozoin sample studied in the main text. The faint slanted-line pattern is due to imperfections in the diamond polishing. Although the raw SEM images are of highenough quality to identify and measure hemozoin crystals, there is some background due to cell residue. We thus postprocess the raw SEM image to enhance hemozoin visibility in Fig. 3(b). The hemozoin crystals are selected with the free selection tool in the graphics editor GIMP, and a mask is created from the selection [Fig. 7(b)]. The mask is used to colorize the selected crystals, and the inverted mask is used to diminish the contrast of the background.

(a)

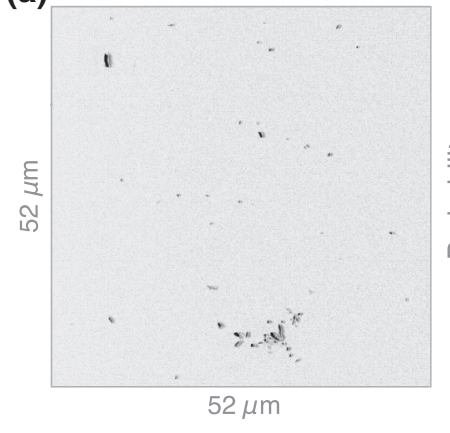

FIG. 8. Size comparison of natural and synthetic hemozoin crystals. (a) SEM image of synthetic hemozoin crystals dispersed on a diamond substrate. (b) Histogram of crystal area for natural and synthetic hemozoin crystals, as determined from SEM images in Figs. 7(a)-7(c) and 8(a). (b)

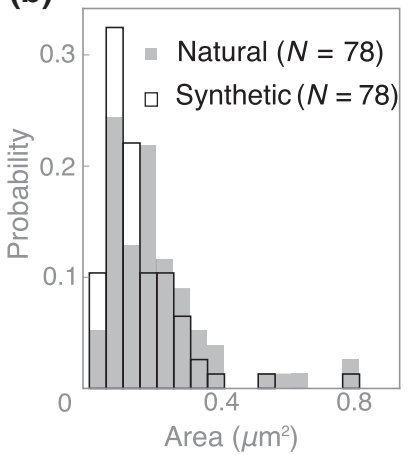


We use another set of 78 synthetic hemozoin crystals [Fig. 8(a)] to characterize the distribution of sizes in the synthetic hemozoin sample. Figure 8(b) shows a histogram of the nanocrystal areas for natural and synthetic hemozoin crystals. The mean area is $0.16 \mu \mathrm{m}^{2}$ for synthetic hemozoin and $0.20 \mu \mathrm{m}^{2}$ for natural hemozoin. The slightly larger size of natural crystals is in agreement with the slightly larger magnetic fields produced by those crystals, as seen in Fig. 5(d).

In the limit that the crystal dimensions are smaller than the magnetic image resolution, we expect the relationship between $\chi$, the mean crystal area $(\bar{A})$, and the mean magnetization slope $\left(\overline{d \Delta B / d B_{0}}\right)$ is $\chi \propto \overline{d \Delta B / d B_{0}} / \bar{A}^{3 / 2}$, where we assume that the mean crystal volume is proportional to $\bar{A}^{3 / 2}$. Using values from Figs. 5(d) and 8(b), we find $\overline{d \Delta B / d B_{0}} / \bar{A}^{3 / 2}=86(\mu \mathrm{T} / \mathrm{T}) / \mu \mathrm{m}^{3 / 2}$ for synthetic crystals

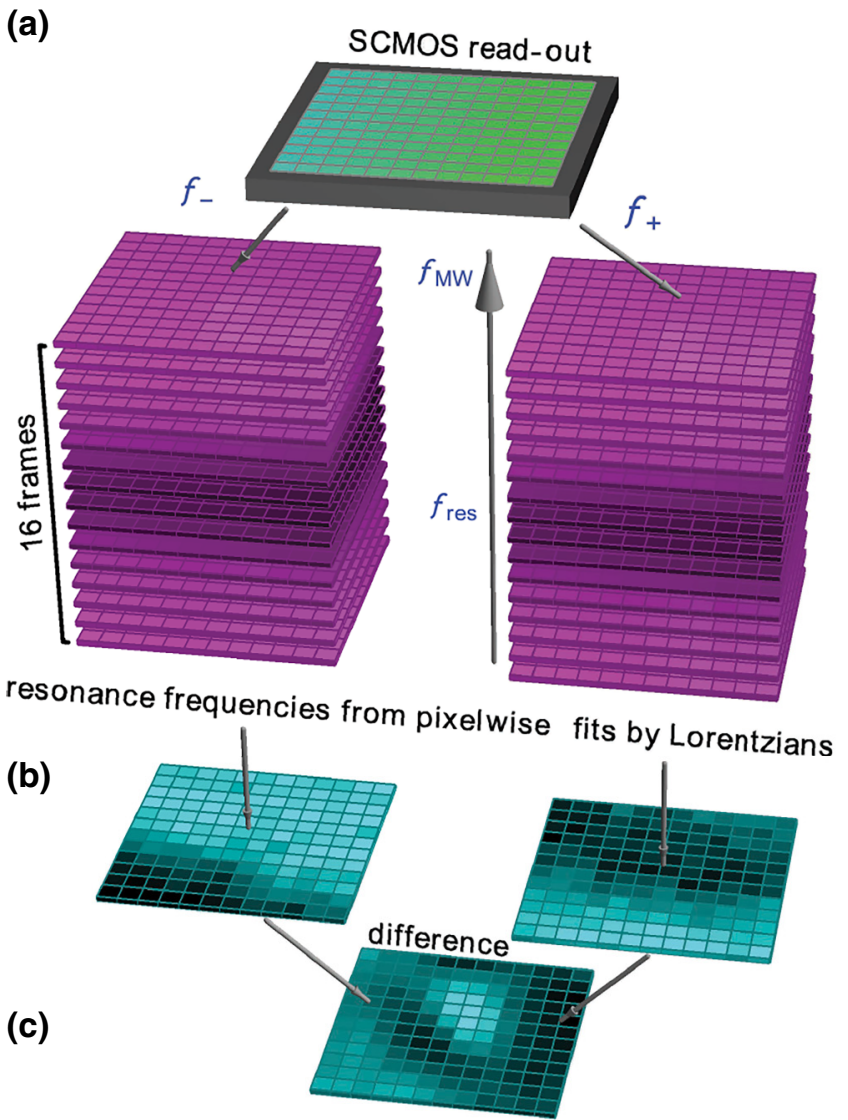

and $90(\mu \mathrm{T} / \mathrm{T}) / \mu \mathrm{m}^{3 / 2}$ for natural crystals. These nearly identical values support our claim that $\chi$ is approximately the same for both populations and that the slightly larger size of natural crystals accounts for their slightly larger magnetic fields.

In Sec. IV, we report that 82 of 120 features identified in the natural-hemozoin SEM images produce observable magnetic features. We also report that 41 of 46 features observed in synthetic hemozoin reflectance images produce observable magnetic features. There are two possible explanations for why some particles do not produce magnetic features: (i) the particles are paramagnetic but we cannot observe their magnetization with the available signal-to-noise ratio or (ii) the particles are actually diamagnetic and we have misidentified them as hemozoin crystals from the SEM (or reflectance) images.

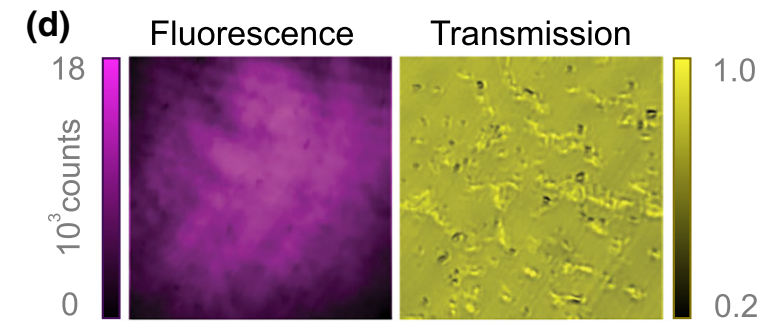

(e)

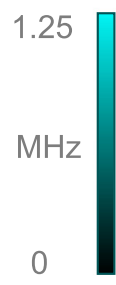

(f)
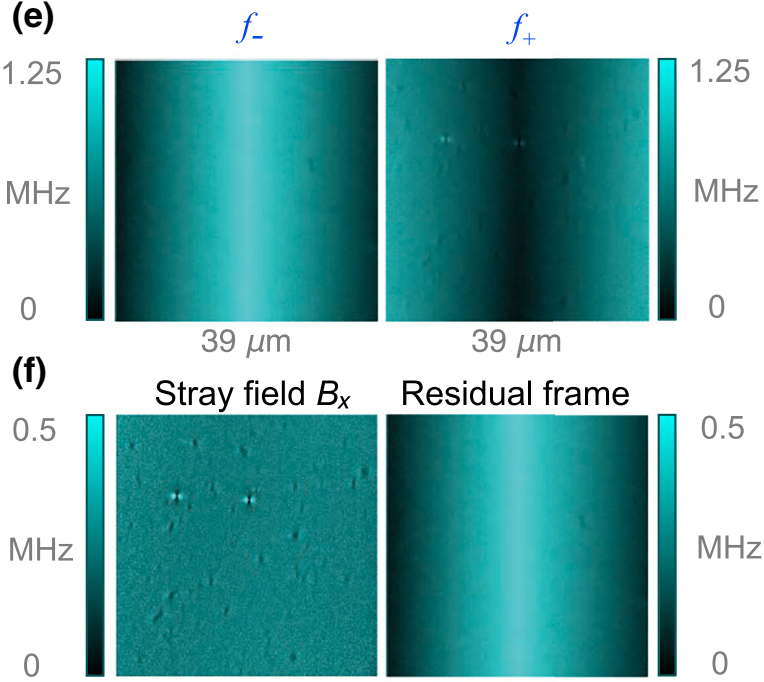

Stray field $B_{X}$

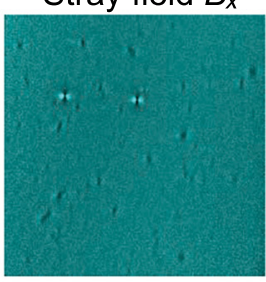

$39 \mu \mathrm{m}$

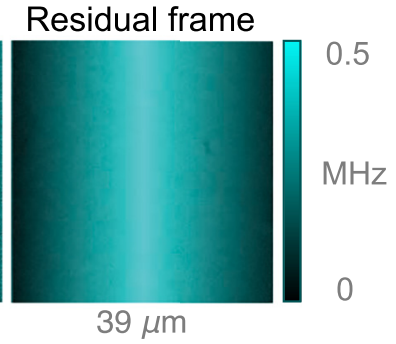

FIG. 9. Data processing. (a) Fluorescence images are acquired at each of 32 different microwave frequencies (16 frequencies for each of the $f_{ \pm}$spin transitions). (b) For any given pixel, two 16-point ODMR curves (fluorescence intensity versus microwave frequency) are generated. Each ODMR curve is fit to a Lorentzian function to reveal the ODMR central frequencies for that pixel. This is repeated for each pixel, yielding two maps of ODMR central frequencies corresponding to $f_{+}$and $f_{-}$. (c) The magnetic field pattern is obtained by subtraction of the images for $f_{+}$and $f_{-}$, division by $2 \gamma_{\mathrm{NV}}$, and subtraction of the applied $B_{0}$ field. (d) An example fluorescence image for a fixed microwave frequency along with a bright-field transmission image of the same field of view. (e) Intermediate step showing separate maps for frequencies $f_{+}$and $f_{-}$. (f) The final map, taken at $B_{0}=350 \mathrm{mT}$, is proportional to stray magnetic fields, $\gamma_{\mathrm{NV}} B_{x}=\left(f_{+}-f_{-}\right) / 2-\gamma_{\mathrm{NV}} B_{0}$. This is shown alongside a map of residual nonmagnetic shifts, $\left(f_{+}+f_{-}\right) / 2-2 D$. Note that $f_{-}$is defined such that it is negative when $|-1\rangle$ is lower in energy than $|0\rangle$. The horizontally varying pattern is an artifact of the sCMOS camera's dual-sensor read-out. This artifact is present when rolling-shutter mode is used, but is eliminated by our subtraction procedure. 
Considering many of these features had an area comparable to, or larger than, the mean crystal area, the first explanation would require that the magnetically inactive crystals have anomalously small volume susceptibility. This is inconsistent with the monolithic hemozoin-crystal structure, which would predict much smaller variations in $\chi$. We therefore conclude that the second explanation is more likely.

\section{APPENDIX D: \\ DIAMOND-MAGNETIC-MICROSCOPY IMAGE PROCESSING}

The principle of our image-processing procedure is shown in Fig. 9. While the microwave frequency is swept in a range of $\sim 12 \mathrm{MHz}$ about each ODMR transition, a set of 32 arrays of fluorescence images are acquired from the sCMOS sensor and accumulated for signal averaging. Each of the images comprises 600 pixels $x$ 600 pixels. Each pixel corresponds to an area of $65 \times$ $65 \mathrm{~nm}^{2}$ in the sample plane [Fig. 9(d)]. The images are arranged in two image stacks with dimensions of $16 \times 600 \times 600$, where the first dimension is arranged by microwave frequency. By fitting Lorentzian functions along the microwave-frequency dimension, we produce two $600 \times 600$ images corresponding to each ODMR central frequency [Figs. 9(b) and 9(e)].

The ODMR central frequency of a particular pixel probed in a particular pixel of the sensor contains information about the local magnetic field, $B_{0}+B_{x}$. However, other factors, such as strain or temperature [37], can also

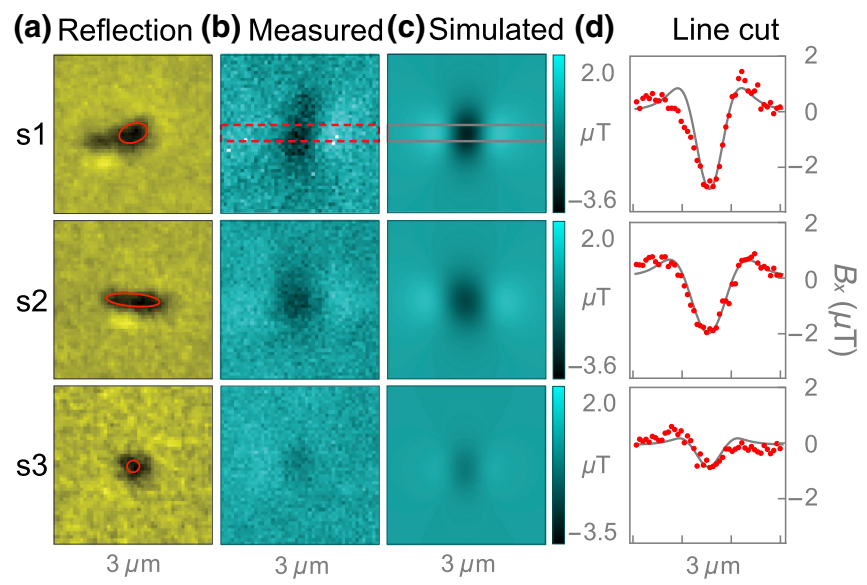

FIG. 10. Individual synthetic hemozoin crystals. (a) Confocal reflection images of hemozoin nanocrystals s1-s3, labeled in Figs. 5(a) and 5(b). The estimated area used for magnetostatic modeling is outlined in red. (b) Corresponding diamondmagnetic-microscopy images $\left(B_{0}=350 \mathrm{mT}\right)$ for each crystal. (c) Simulated magnetic images $\left(\chi=3.4 \times 10^{-4}, B_{0}=350 \mathrm{mT}\right)$ obtained with nanocrystal dimensions roughly estimated from (a). (d) Line cuts of each nanocrystal (red, measured; gray, simulated), from which the field pattern amplitude $\Delta B$ is inferred. produce frequency shifts. Fortunately, the difference of the two ODMR frequencies gives a pure measure of the magnetic field, $f_{+}-f_{-}=2 \gamma_{\mathrm{NV}}\left(B_{0}+B_{x}\right)$. Subtraction of the contribution from $B_{0}$ gives the final magnetic images as in Figs. 9(c) and 9(f). The horizontally varying pattern in Fig. 9(e) is actually varying along columns of the sCMOS sensor (the images are rotated by $90^{\circ}$ in our setup). This is an artifact of using the sCMOS sensor's rolling -shutter mode. The sCMOS camera has two sensors, and the read-out from each sensor starts from the center rows and moves out to the edges of the sCMOS chip. The total read-out time is $\sim 3 \mathrm{~ms}$, which is approximately the same as the exposure time. This means that rows in the center see a microwave frequency different from that seen by the rows at the edges. The difference is approximately the sweep range divided by the number of microwave points, $12 \mathrm{MHz} / 16=0.75 \mathrm{MHz}$. To eliminate this artifact, we scan the microwave frequencies across both ODMRs in the same direction for $B_{0}<102.5 \mathrm{mT}$ ( $f_{+}$and $f_{-}$are both positive), whereas for $B_{0}>102.5 \mathrm{mT}$ ( $f_{+}$and $f_{-}$have opposite signs) we scan the microwave frequencies in opposite directions. This scanning and subtraction procedure is effective in removing this artifact [Fig. 9(f)].

We use 16 frequencies per ODMR peak, as we find that near-shot-noise-limited performance can be realized in this manner without introducing artifacts. This leads to a maximum magnetic image acquisition rate of $\sim 10 \mathrm{~Hz}$. While we have performed Lorentzian fitting off-line, if one have to implement this postprocessing procedure in real time, the image rate may be further reduced. However, in principle, only two frequencies per resonance are necessary, and in this manner the ODMR positions could be determined algebraically without Lorentzian fitting. This would allow magnetic image refresh rates of $\sim 100 \mathrm{~Hz}$, enabling video recording of fast dynamics.

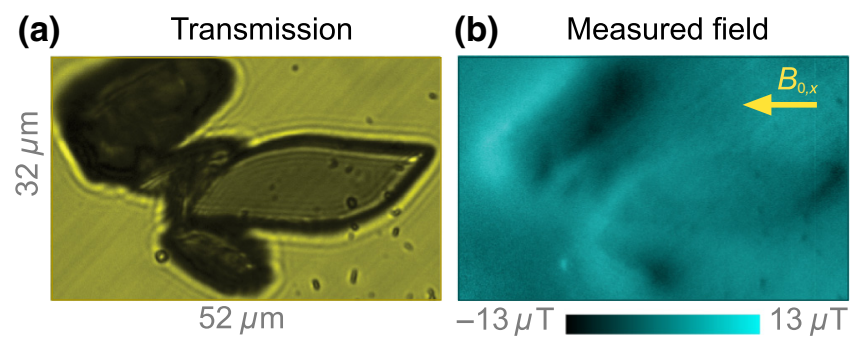

FIG. 11. Magnetic imaging of hemin crystals. (a) Brightfield transmission image of hemin crystals. (b) Corresponding diamond-magnetic-microscopy image $\left(B_{0}=186 \mathrm{mT}\right)$. The inplane component of the applied field is labeled with an arrow. An out-of-plane component of similar magnitude is also present. Smaller particles are synthetic hemozoin crystals that are dispersed along with hemin crystals. 


\section{APPENDIX E: AUXILIARY RESULTS}

\section{Magnetostatic modeling of $s 1-s 3$}

Three individual crystals, labeled s1-s3 in the images in Figs. 5(b) and 5(c), are addressed in detail in Fig. 10. Figure 10(a) shows confocal reflection images of each crystal. Figure 10(b) shows the corresponding diamond-magnetic-microscopy images taken at $B_{0}=$ $186 \mathrm{mT}$. As with the natural hemozoin sample shown in Figs. 4(a)-4(c), each synthetic crystal exhibits a different field pattern characteristic of its unique size, shape, and orientation.

Figure 10(c) shows the expected magnetic field patterns of each nanocrystal, calculated using the procedure described in Sec. II. Each nanocrystal is modeled as a 3D ellipsoid with uniform susceptibility and dimensions roughly estimated from the corresponding confocal reflection images. The height of the crystals is assumed to be $200 \mathrm{~nm}$. For all crystals, the model produces similar field patterns to those observed experimentally. The pattern amplitude is best described using a susceptibility of $\chi=3.4 \times 10^{-4}$, which is the same as the value of $\chi$ used for natural hemozoin crystals (Sec. IV).

Line cuts of the magnetic field patterns for s1-s3 are shown in Fig. 10(d). The line cuts are obtained by averaging $B_{x}$ values over six rows $(390 \mathrm{~nm})$ in a band along the magnetic feature, as indicated in Figs. 10(b) and $10(\mathrm{c})$. The line cuts are used to calculate the magnetic pattern amplitudes $\Delta B$ at each value of $B_{0}$. The results of this analysis are displayed in Figs. 5(c) and 5(d) and 14.

\section{Diamond magnetic microscopy of hemin}

Our diamond magnetic microscope is capable of imaging paramagnetic substances other than hemozoin. Of possible interest are hemin microcrystals, which are used as a pharmacological agent for treating porphyria [56]. Hemin crystals have magnetic susceptibility similar to that of hemozoin but are much larger (up to $30 \mu \mathrm{m}$ ).

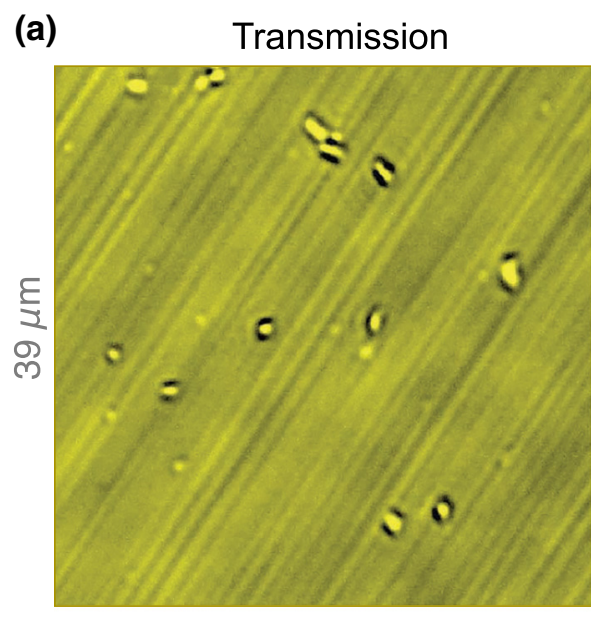

0.2

(d)

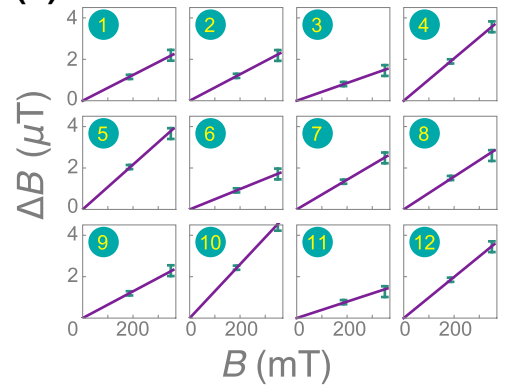

(b)

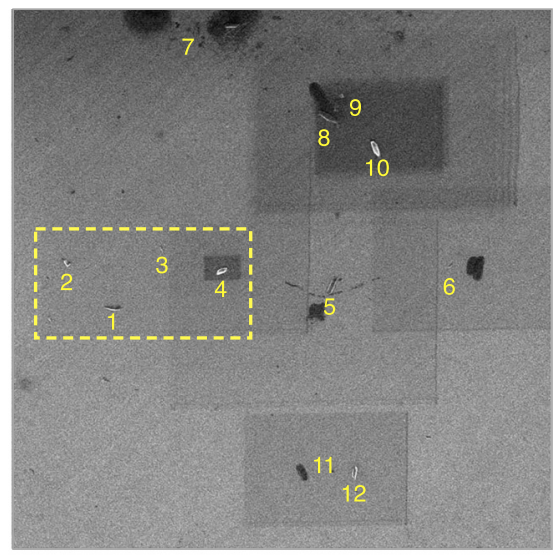

$39 \mu \mathrm{m}$ (c) $\quad$ Measured $B_{x}$

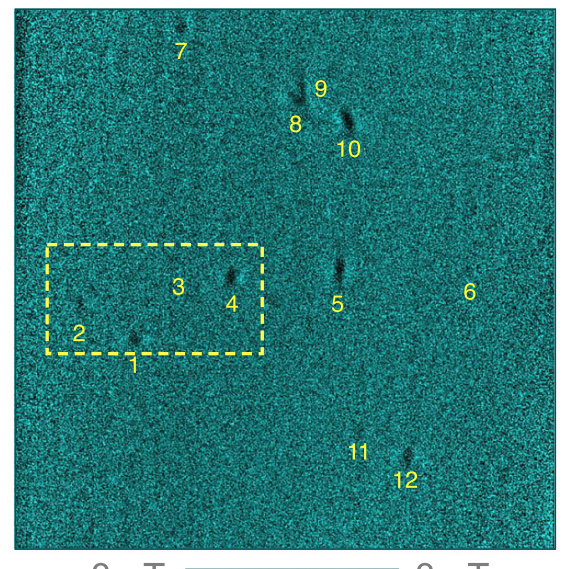

(e)

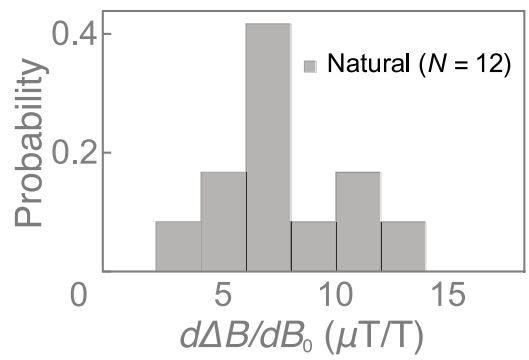

(f)

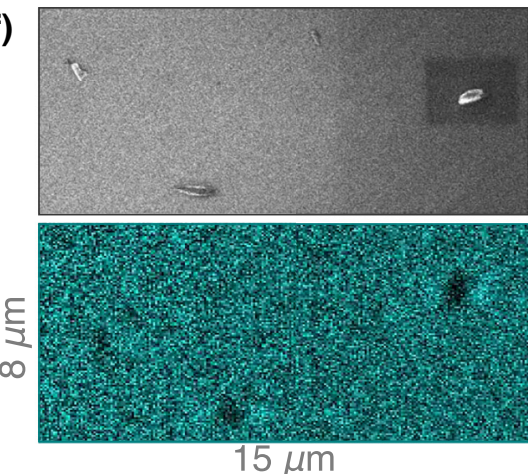

FIG. 12. A second region of the natural hemozoin sample. (a) Bright-field transmission image of natural hemozoin crystals dispersed on a diamond substrate. (b) SEM image of the same nanocrystals. Dark rectangles are due to overcharging during zooming. (c) Corresponding diamond-magnetic-microscopy image at $B_{0}=350 \mathrm{mT}$ showing the positions of each nanocrystal. (d) $\Delta B\left(B_{0}\right)$ curves for all 12 hemozoin crystals. (e) Histogram of $d \Delta B / d B_{0}$ determined from the linear slopes in (e). (f) Magnified SEM and magnetic images of crystals $1-4$. 

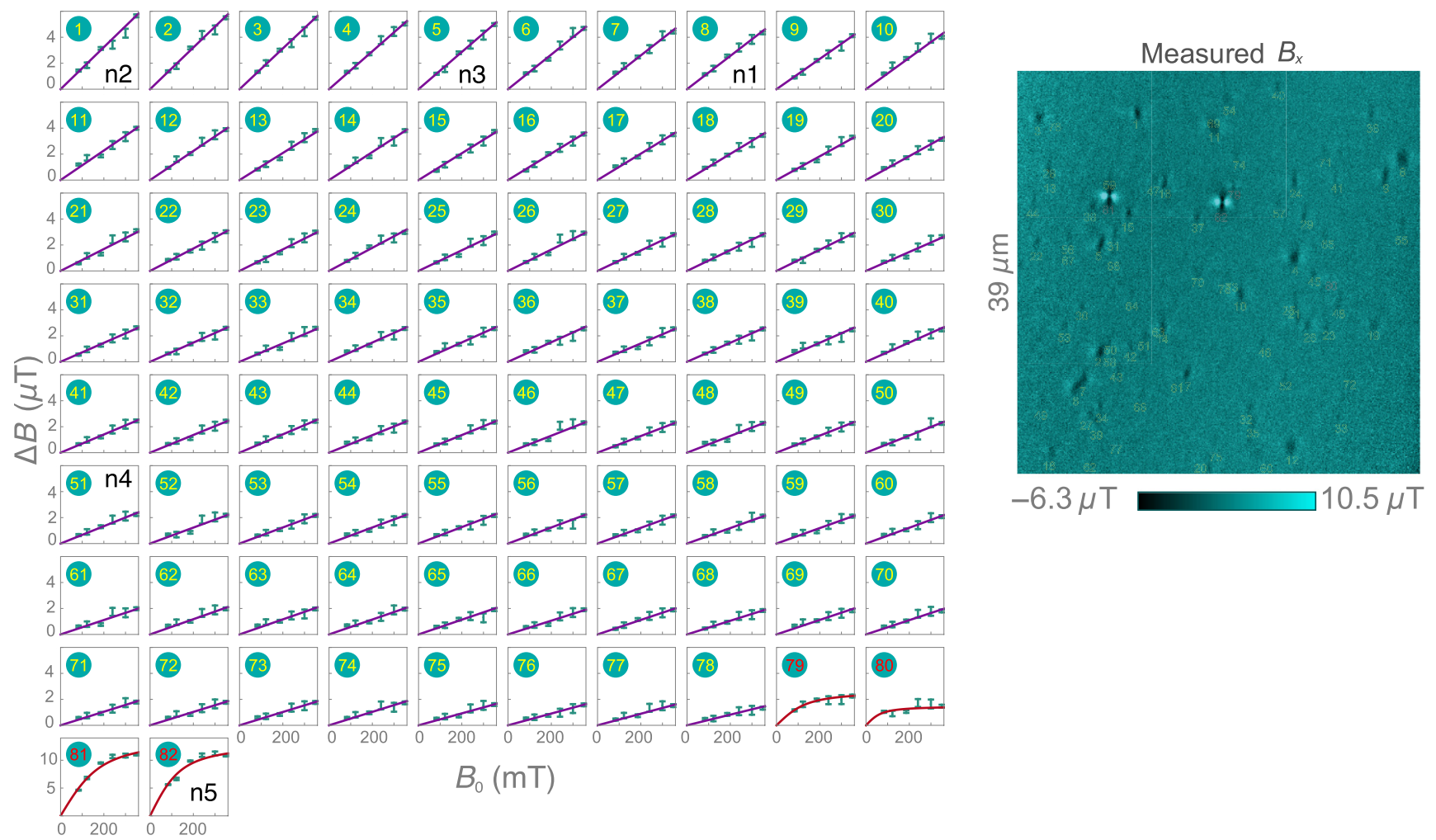

FIG. 13. Natural hemozoin: field-dependent magnetization. $\Delta B\left(B_{0}\right)$ curves for all 82 natural hemozoin crystals reported in Sec. IV (left). Magnetic image $\left(B_{0}=350 \mathrm{mT}\right)$ showing the positions of each nanocrystal (right).

They thus serve as a convenient test sample for imaging paramagnetism.

\section{Natural hemozoin in a second field of view}

In addition to the hemozoin samples discussed in Sec. IV, we have also studied natural hemozoin crystals in a second region of the diamond substrate. Figures 12(a)-12(c) show, respectively, bright-field transmission,
SEM, and diamond-magnetic-microscopy images in this second field of view. The hemozoin density is sparser in this region (only 12 crystals are identified) and less cell residue is present. Figure 12(d) shows the $\triangle B\left(B_{0}\right)$ curves for each of the 12 crystals, and Fig. 12(e) shows the histogram of the fitted slopes. The distribution is similar to that for natural hemozoin shown in Fig. 5(d). Figure 12(f) shows a magnified view of the SEM and magnetic images containing four crystals.
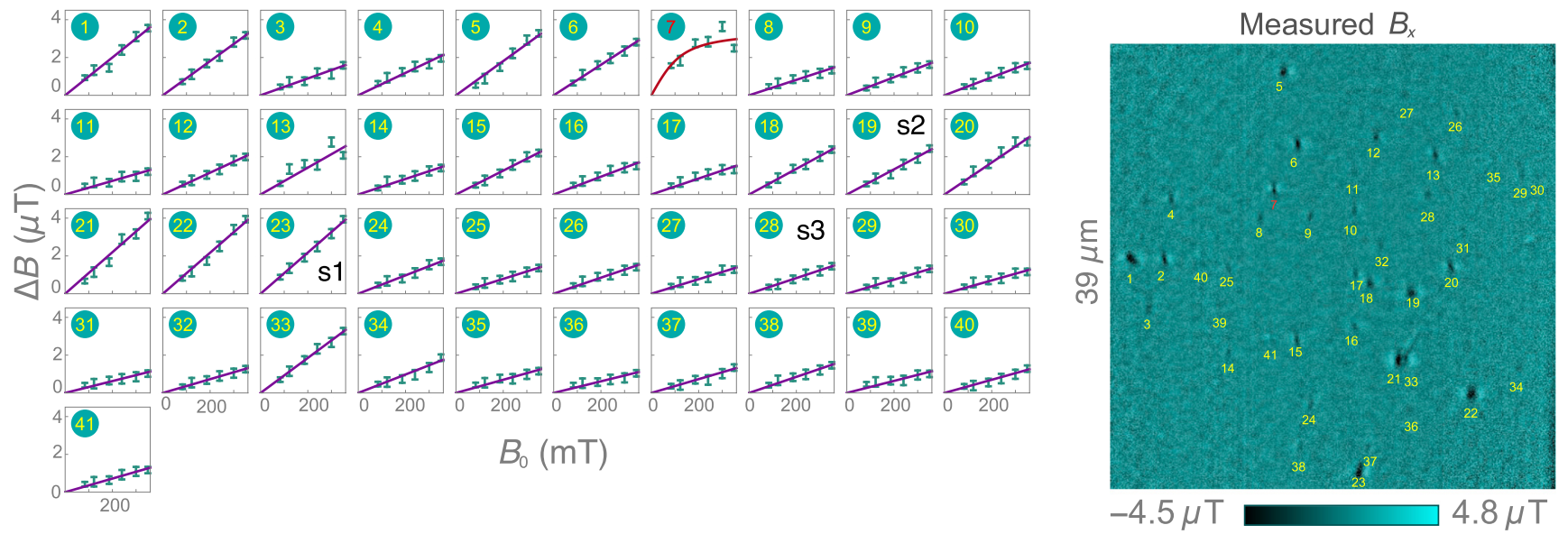

FIG. 14. Synthetic hemozoin: field-dependent magnetization. $\Delta B\left(B_{0}\right)$ curves for all 41 synthetic hemozoin crystals reported in Sec. IV (left). Magnetic image $\left(B_{0}=350 \mathrm{mT}\right)$ showing the positions of each nanocrystal (right). 


\section{Complete field-dependent magnetization data set for samples studied in Sec. IV}

Figure 13 shows $\Delta B\left(B_{0}\right)$ curves for all 82 observed magnetic features in the natural hemozoin sample. The paramagnetic crystals are labeled with yellow numbers, while particles exhibiting superparamagneticlike behavior are labeled with red numbers. The corresponding magnetic features are labeled with numbers on the wide-field magnetic image (taken at $B_{0}=350 \mathrm{mT}$ ).

Figure 14 shows $\Delta B\left(B_{0}\right)$ curves for the 41 magnetic features observed in the synthetic hemozoin sample reported in Sec. IV. The particle exhibiting superparamagnetic behavior is labeled with a red number. The corresponding magnetic features are labeled with numbers on the wide-field magnetic image (taken at $B_{0}=350 \mathrm{mT}$ ).

Figure 11(a) shows a bright-field transmission image of hemin crystals dispersed on a diamond substrate. Figure 11(b) shows the corresponding diamond magnetic image. The magnetic pattern of these crystals appears different from the field patterns presented elsewhere in this paper. This is because we have used a different diamond substrate (with a $1.7-\mu \mathrm{m}$-thick NV layer) that is polished with [100] surfaces. All four NV axes in this substrate are directed at $55^{\circ}$ from the surface normal, and the magnetic field is applied along one of these NV axes. This geometry leads to more asymmetric patterns than the in-plane field geometry used elsewhere. The pattern amplitudes are consistent with a magnetic susceptibility $\chi \approx 3 \times 10^{-4}$, similar to that of hemozoin. Some smaller particles shown on Fig. 11 are hemozoin crystals measured along with hemin crystals.

\section{APPENDIX F: UNCERTAINTY IN SUSCEPTIBILITY}

For both natural and synthetic hemozoin, we estimate a relative uncertainty in $\chi$ of $\sim 25 \%$ or less due to imperfect assumptions on NV-layer distribution, image blur, and hemozoin crystal dimensions and shape (see Fig. 15). The image blur is incorporated by convolving the magnetic image with a two-dimensional Gaussian kernel. Some of these assumptions are conflated; for example, image blur and crystal height both contribute to the width of the line cuts seen in Figs. 4(d) and 10(d). We find the best agreement when using a blur with a FWHM of $540 \mathrm{~nm}$ and a crystal height of $200 \mathrm{~nm}$. However, we have observe some magnetic features with a FWHM of $400 \mathrm{~nm}$, such as n5 in Fig. 4(d), which is closer to the expected diffractionlimited resolution of our microscope $(\sim 390 \mathrm{~nm})$. This suggests either the image blur is spatially varying (due to imaging aberrations) or the extra modeled blur is compensating imperfections in another assumption, such as crystal height. We find that reducing the image blur by $15 \%$ [Fig. 15(b)] leads to a best-fit estimate of $\chi=2.7 \times 10^{-4}$, which is $21 \%$ smaller than the estimate made in Sec. IV. (a)

Initial parameters for crystal n4

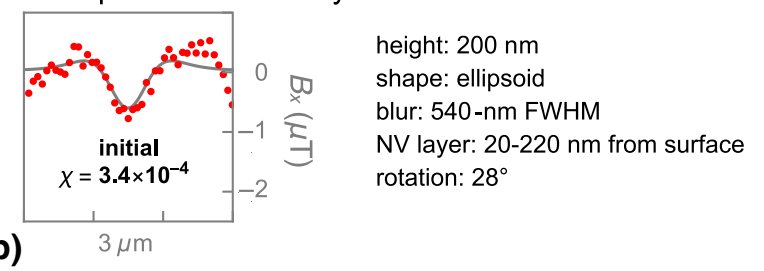

Variation of model parameters
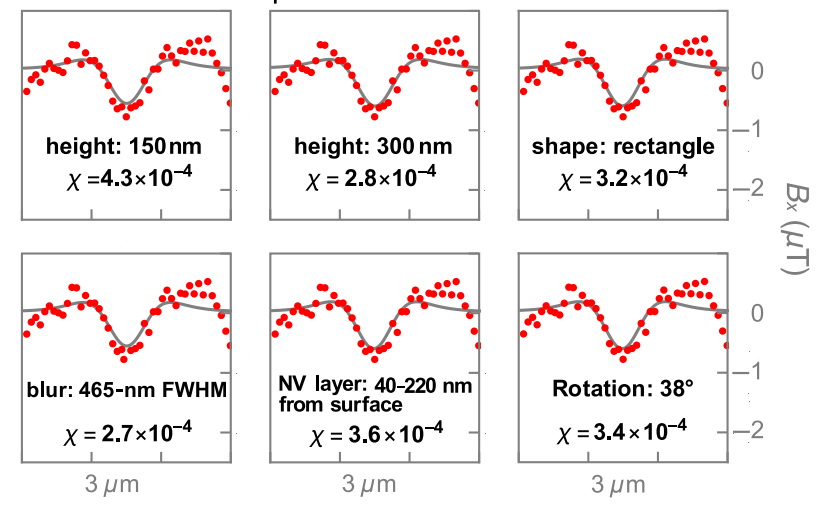

FIG. 15. Impact of model parameters on magnetic susceptibility $\chi$. (a) Line cuts of measured (red) and simulated (gray) magnetic patterns from crystal n4 [reproduced from Fig. 4(d)] obtained with the initial model parameters shown on the right. (b) Simulated line cuts of n 4 for different model parameters. Only one parameter is changed at a time. The varied model parameters and best-fit magnetic susceptibility values are shown as insets.

However, the overall agreement with use of those parameters is slightly worse than for the assumptions of 540-nm blur and $\chi=3.4 \times 10^{-4}$ made in Sec. IV. By comparing the best-fit values of $\chi$ under various reasonable perturbations to the model, we arrive at an uncertainty in $\chi$ of $\sim 25 \%$ or less.

Another source of systematic uncertainty is that we treat $\chi$ as an isotropic quantity. Two factors suggest $\chi$ is actually anisotropic [18]: (i) the crystals are elongated, and therefore may be more susceptible to magnetize along their long axes, and (ii) the iron centers in hematin dimers have $C_{4 v}$ symmetry [Fig. 2(c)], which is largely preserved after crystal formation. The latter suggests a hard axis and an easy plane, whereas the former suggests all three components of magnetization may be different. Butykai et al. [18] made the hard-axis and easy-plane assumption and found a magnetic anisotropy $M_{\text {hard axis }} / M_{\text {easy plane }}$ of $1.16 \pm 0.03$ at room temperature. We have not incorporated magnetic anisotropy in our model because we have not directly measured it and we do not have information about crystal orientation. However, the variation in $\chi$ due to anisotropy is smaller than the estimated uncertainty due to imperfect model assumptions. Nonetheless, it may be a contributing factor to the slight disagreement for some crystals, such as n2 [see Fig. 4(d)]. None of these uncertainties come close to being large enough to explain the 
TABLE I. Methods for label-free imaging of individual hemozoin crystals.

\begin{tabular}{llll}
\hline \hline Imaging method & Physical observables & Penetration of probe & Frame rate (Hz) \\
\hline Absorption microscopy [57] & Optical absorption & Optical field & $\sim 100$ \\
Polarization microscopy [58] & Birefringence or dichroism & Optical field & $\sim 100$ \\
Differential interference contrast [59] & Refractive index & Optical field & $\sim 50$ \\
Resonance Raman microscopy [60] & Vibrational structure & Intense optical field & $\sim 1$ \\
Third-harmonic generation [17] & Crystal structure & Intense optical field & $\sim 1$ \\
Diamond magnetic microscopy (this study) & Magnetic moment & dc or ac magnetic field & $\sim 1$ \\
\hline \hline
\end{tabular}

behavior of $\mathrm{n} 5$ or the other presumed superparamagnetic nanoparticles.

\section{APPENDIX G: SUPERPARAMAGNETISM}

Several points are made in Sec. IV that support the argument that five outlier nanoparticles (including n5) are superparamagnetic. Here we outline four more pieces of evidence supporting this argument:

(a) The nanoparticles are small. For two of the features we do not see anything in the SEM images at the locations of the magnetic features. The other features do have small corresponding particles in their SEM (or confocal reflectance) images, but we cannot be sure they are responsible for the magnetic features.

(b) All five particles have zero coercivity; that is, $\Delta B(0)=0$. Thus, they are unlikely to be ferromagnetic.

(c) The magnetic patterns of these particles are always aligned in the same way as the features from the linear paramagnetic crystals. This further decreases the likelihood these particles are ferromagnetic since they appear to be magnetized by the applied field even at fields below $0.1 \mathrm{~T}$.

(d) The magnetizing field (the $B_{0}$ field needed to magnetize the particles to half their maximum saturation magnetization) is $\sim 0.1 \mathrm{~T}$. This is a reasonable value for superparamagnetic particles [61].

\section{APPENDIX H: COMPARISON WITH OTHER IMAGING METHODS}

Several techniques capable of label-free imaging of individual hemozoin crystals are compared in Table I. Optical methods such as absorption microscopy, polarization microscopy, and differential interference microscopy use weak optical fields and are able to register high-contrast images of hemozoin at fast frame rates $(\sim 100 \mathrm{~Hz})$. Resonance Raman microscopy and third-harmonic-generation imaging use more-invasive focused laser beams and require slower scanning, but are capable of visualizing hemozoin with a high degree of specificity. In contrast, diamond magnetic microscopy does not require penetration of light into the hemozoin sample [55] and is the only method capable of registering quantitative magnetic images of individual hemozoin crystals. The frame rate of our method of $\sim 1 \mathrm{~Hz}$ is slower than that of the weak optical probe methods but comparable to that of third-harmonic-generation imaging and resonance Raman microscopy.

The perturbative nature of diamond magnetic microscopy lies in the application of weak microwave pulses and a large dc-bias magnetic field. The microwave field could, in principle, lead to heating of the sample. We typically use a low-enough microwave power that the diamond temperature increases by $\sim 2{ }^{\circ} \mathrm{C}$ or less, as measured by the shift in NV ODMR [37]. Considering diamond's excellent thermal properties, we can expect that the samples will undergo an even-smaller temperature change. Diamond magnetic microscopy has already been successfully applied to the study of living cells $[3,5,62,63]$. It is not known whether the introduction of a large bias field may impact hemozoin formation processes in red blood cells. If this is found to be invasive, a time-lapse approach may be required where the field is off for long periods followed by short measurement intervals.

[1] Romana Schirhagl, Kevin Chang, Michael Loretz, and Christian L. Degen, Nitrogen-vacancy centers in diamond: Nanoscale sensors for physics and biology, Annu. Rev. Phys. Chem. 65, 83 (2014).

[2] Loïc Rondin, Jean-Philippe Tetienne, Thomas Hingant, Jean-François Roch, Patrick Maletinsky, and Vincent Jacques, Magnetometry with nitrogen-vacancy defects in diamond, Rep. Prog. Phys. 77, 056503 (2014).

[3] David Le Sage, Koji Arai, David R. Glenn, Stephen J. De Vience, Linh M. Pham, Lilah Rahn-Lee, Mikhail D. Lukin, Amir Yacoby, Arash Komeili, and Ronald L. Walsworth, Optical magnetic imaging of living cells, Nature 496, 486 (2013).

[4] Florestan C. Ziem, Nicolas S. Götz, Andrea Zappe, Steffen Steinert, and Jörg Wrachtrup, Highly sensitive detection of physiological spins in a microfluidic device, Nano Lett. 13, 4093 (2013).

[5] David R. Glenn, Kyungheon Lee, Hongkun Park, Ralph Weissleder, Amir Yacoby, Mikhail D. Lukin, Hakho Lee, Ronald L. Walsworth, and Colin B. Connolly, Single-cell magnetic imaging using a quantum diamond microscope, Nat. Methods 12, 736 (2015). 
[6] John F. Barry, Matthew J. Turner, Jennifer M. Schloss, David R. Glenn, Yuyu Song, Mikhail D. Lukin, Hongkun Park, and Ronald L. Walsworth, Optical magnetic detection of single-neuron action potentials using quantum defects in diamond, Proc. Natl. Acad. Sci. U. S. A. 113, 14133 (2016).

[7] Michael Gould, Russell J. Barbour, Nicole Thomas, Hamed Arami, Kannan M. Krishnan, and Kai-Mei C. Fu, Roomtemperature detection of a single $19 \mathrm{~nm}$ super-paramagnetic nanoparticle with an imaging magnetometer, Appl. Phys. Lett. 105, 072406 (2014).

[8] Jean-Philippe Tetienne, Alain Lombard, David A. Simpson, Cameron Ritchie, Jianing Lu, Paul Mulvaney, and Lloyd C. L. Hollenberg, Scanning nanospin ensemble microscope for nanoscale magnetic and thermal imaging, Nano. Lett. 16, 326 (2016).

[9] Hunter C. Davis, Pradeep Ramesh, Aadyot Bhatnagar, Audrey Lee-Gosselin, John F. Barry, David R. Glenn, Ronald L. Walsworth, and Mikhail G. Shapiro, Mapping the microscale origins of MRI contrast with subcellular NV diamond magnetometry, Nat. Commun. 9, 131 (2018).

[10] Lorena M. Coronado, Christopher T. Nadovich, and Carmenza Spadafora, Malarial hemozoin: From target to tool, Biochim. Biophys. Acta 1840, 2032 (2014).

[11] David J. Sullivan, Theories on malarial pigment formation and quinoline action, Int. J. Parasitol. 32, 1645 (2002).

[12] Ernst Hempelmann, Hemozoin biocrystallization in Plasmodium falciparum and the antimalarial activity of crystallization inhibitors, Parasitol. Res. 100, 671 (2007).

[13] Sangyeon Cho, Soomin Kim, Youngchan Kim, and YongKeun Park, Optical imaging techniques for the study of malaria, Trends Biotechnol. 30, 71 (2012).

[14] Maria Rebelo, Claudia Sousa, Howard M. Shapiro, Maria M. Mota, Martin P. Grobusch, and Thomas Hänscheid, A novel flow cytometric hemozoin detection assay for realtime sensitivity testing of Plasmodium falciparum, PLoS ONE 8, e61606 (2013).

[15] Christine Lawrence and Julie A. Olson, Birefringent hemozoin identifies malaria, Am. J. Clin. Pathol. 86, 360 (1986).

[16] Rosangela Frita, Maria Rebelo, Ana Pamplona, Ana M. Vigario, Maria M. Mota, Martin P. Grobusch, and Thomas Hänscheid, Simple flow cytometric detection of haemozoin containing leukocytes and erythrocytes for research on diagnosis, immunology and drug sensitivity testing, Malaria J. 10, 74 (2011).

[17] Jonathan M. Bélisle, Santiago Costantino, Mara L. Leimanis, Marie-Josée Bellemare, D. Scott Bohle, Elias Georges, and Paul W. Wiseman, Sensitive detection of malaria infection by third harmonic generation imaging, Biophys. J. 94, L26 (2008).

[18] Adam Butykai, Agnes Orbán, Viktória Kocsis, Dávid Szaller, Sándor Bordács, Erzsébet Tátrai-Szekeres, Levente Kiss, Attila Bóta, Beáta Vértessy, Tivadar Zelles, and Istvan Kézsmárki, Malaria pigment crystals as magnetic microrotors: Key for high-sensitivity diagnosis, Sci. Rep. 3, 1431 (2013).

[19] Agnes Orban, Maria Rebelo, Petra Molnar, Inês S. Albuquerque, Adam Butykai, and Istvan Kezsmarki, Efficient monitoring of the blood-stage infection in a malaria rodent model by the rotating-crystal magneto-optical method, Sci. Rep. 6, 23218 (2016).
[20] Samantha E. McBirney, Dongyu Chen, Alexis Scholtz, Hossein Ameri, and Andrea M. Armani, Rapid diagnostic for point-of-care malaria screening, ACS Sens. 3, 1264 (2018).

[21] Weng Kung Peng, Tian Fook Kong, Chee Sheng Ng, Lan Chen, Yongxue Huang, Ali Asgar S. Bhagat, Nam Trung Nguyen, Peter Rainer Preiser, and Jongyoon Han, Micromagnetic resonance relaxometry for rapid label-free malaria diagnosis, Nat. Med. 20, 1069 (2014).

[22] Yves Gossuin, Philippe Okusa Ndjolo, Quoc Lam Vuong, and Pierre Duez, NMR relaxation properties of the synthetic malaria pigment $\beta$-hematin, Sci. Rep. 7, 14557 (2017).

[23] Andrzej Sienkiewicz, J. Krzystek, Bertrand Vileno, Guillaume Chatain, Aaron J. Kosar, D. Scott Bohle, and László Forró, Multi-frequency high-field EPR study of iron centers in malarial pigments, J. Am. Chem. Soc. 128, 4534 (2006).

[24] Charles C. Kim, Emily B. Wilson, and Joseph L. Derisi, Improved methods for magnetic purification of malaria parasites and haemozoin, Malar. J. 9, 17 (2010).

[25] Sara Lyons Hackett, Juliana Hamzah, Timothy M. E. Davis, and Timothy G. St. Pierre, Magnetic susceptibility of iron in malaria-infected red blood cells, Biochim. Biophys. Acta 1792, 93 (2009).

[26] Claude Brémard, Girerd Jean Jacques, Jean Claude Merlin, and Serge Moreau, Iron (iii) porphyrin aggregates grafted on agarose gel as models of hemoglobin degradation products, J. Mol. Struct. 267, 117 (1992).

[27] D. Scott Bohle, Peter Debrunner, Peter A. Jordan, Sara K. Madsen, and Charles E. Schulz, Aggregated heme detoxification byproducts in malarial trophozoites: $\beta$-hematin and malaria pigment have a single $S=5 / 2$ iron environment in the bulk phase as determined by EPR and magnetic Mössbauer spectroscopy, J. Am. Chem. Soc. 120, 8255 (1998).

[28] Linh M. Pham, David Le Sage, Paul L. Stanwix, Tai Kong Yeung, Derek W. Glenn, Andrey Trifonov, Paola Cappellaro, Philip R. Hemmer, Mikhail D. Lukin, Hongsik Park, Amir Yacoby, and Ronald L. Walsworth, Magnetic field imaging with nitrogen-vacancy ensembles, New J. Phys. 13, 045021 (2011).

[29] Jean-Philippe Tetienne, Nikolai Dontschuk, David A. Broadway, Alastair Stacey, David A. Simpson, and Lloyd C. L. Hollenberg, Quantum imaging of current flow in graphene, Sci. Adv. 3, e1602429 (2017).

[30] Victor M. Acosta, Erik Bauch, Micah P. Ledbetter, Charles Santori, Kai-Mei C. Fu, Paul E. Barclay, Raymond G. Beausoleil, Héloïse Linget, Jean Francois Roch, Francois Treussart, Sergey D. Chemerisov, Wojciech Gawlik, and Dmitry Budker, Diamonds with a high density of nitrogenvacancy centers for magnetometry applications, Phys. Rev. B 80, 115202 (2009).

[31] E. Ed Kleinsasser, Matthew M. Stanfield, Jannel K. Q. Banks, Zhouyang Zhu, Wen-Di Li, Victor M. Acosta, Hideyuki Watanabe, Kohei M. Itoh, and Kai-Mei C. Fu, High density nitrogen-vacancy sensing surface created via $\mathrm{He}^{+}$ion implantation of ${ }^{12} \mathrm{C}$ diamond, Appl. Phys. Lett. 108, 202401 (2016).

[32] V. M. Acosta, E. Bauch, A. Jarmola, L. J. Zipp, M. P. Ledbetter, and D. Budker, Broadband magnetometry by 
infrared-absorption detection of nitrogen-vacancy ensembles in diamond, Appl. Phys. Lett. 97, 174104 (2010).

[33] Kejie Fang, Victor M. Acosta, Charles Santori, Zhihong Huang, Kohei M. Itoh, Hideyuki Watanabe, Shinichi Shikata, and Raymond G. Beausoleil, High-sensitivity magnetometry based on quantum beats in diamond nitrogen-vacancy centers, Phys. Rev. Lett. 110, 130802 (2013).

[34] Brendan J. Shields, Quirin P. Unterreithmeier, Nathalie P. de Leon, Hee Su Park, and Mikhail D. Lukin, Efficient readout of a single spin state in diamond via spin-to-charge conversion, Phys. Rev. Lett. 114, 136402 (2015).

[35] Vladimir Dolgovskiy, Ilja Fescenko, Noboru Sekiguchi, Simone Colombo, Victor Lebedev, Jiawan Zhang, and Antoine Weis, A magnetic source imaging camera, Appl. Phys. Lett. 109, 023505 (2016).

[36] Pauli Kehayias, Andrey Jarmola, Nazanin Mosavian, Ilja Fescenko, Francisco M. Benito, Abdelghani Laraoui, Janis Smits, Lykourgos Bougas, Dmitry Budker, Alexander Neumann, Steve R. J. Brueck, and Victor M. Acosta, Solution nuclear magnetic resonance spectroscopy on a nanostructured diamond chip, Nat. Commun. 8, 188 (2017).

[37] Victor M. Acosta, Erik Bauch, Micah P. Ledbetter, Amir Waxman, Louis S. Bouchard, and Dmitry Budker, Temperature dependence of the nitrogen-vacancy magnetic resonance in diamond, Phys. Rev. Lett. 104, 070801 (2010).

[38] Erik Bauch, Connor A. Hart, Jennifer M. Schloss, Matthew J. Turner, John F. Barry, Pauli Kehayias, Swati Singh, and Ronald L. Walsworth, Ultralong dephasing times in solidstate spin ensembles via quantum control, Phys. Rev. X 8, 031025 (2018).

[39] Michail Inyushin, Yuriy V. Kucheryavih, Lilia Y. Kucheryavih, Luévanos Rojas, Igor Khmelinskii, and Vladimir I. Makarov, Superparamagnetic properties of hemozoin, Sci. Rep. 6, 26212 (2016).

[40] William T. Coffey and Yuri P. Kalmykov, in The Langevin Equation (Publisher World Scientific, 2017), Vol. 28, Chap. 1, p. 1.

[41] Igor Khmelinskii and Vladimir I. Makarov, Temperature dependence of the spin relaxation time of $\mathrm{Fe}_{3} \mathrm{O}_{4}$ and hemozoin superparamagnetic nanocrystals, Chem. Phys. 493, 120 (2017).

[42] Maritza Jaramillo, Marie Josée Bellemare, Caroline Martel, Marina Tiemi Shio, Ana Paulina Contreras, Marianne Godbout, Michel Roger, Eric Gaudreault, Jean Gosselin, D. Scott Bohle, and Martin Olivier, Synthetic Plasmodiumlike hemozoin activates the immune response: A morphology-function study, PLoS ONE 4, e6957 (2009).

[43] Marcus F. Oliveira, Stefan W. Kycia, Ariel Gomez, Aaron J. Kosar, D. Scott Bohle, Ernst Hempelmann, Diego Menezes, Marcos André Vannier-Santos, Pedro L. Oliveira, and Sérgio T. Ferreira, Structural and morphological characterization of hemozoin produced by Schistosoma mansoni and Rhodnius prolixus, FEBS Lett. 579, 6010 (2005).

[44] Ágnes Orbán, Ádám Butykai, András Molnár, Zófia Prähle, Gergö Fülöp, Tivadar Zelles, Wasan Forsyth, Danika Hill, Ivo Müller, Louis Schofield, Maria Rebelo, Thomas Hänscheid, Stephan Karl, and István Kézsmárki, Evaluation of a novel magneto-optical method for the detection of malaria parasites, PLoS ONE 9, e96981 (2014).
[45] Viktor Stepanov, Franklin H. Cho, Chathuranga Abeywardana, and Susumu Takahashi, High-frequency and highfield optically detected magnetic resonance of nitrogenvacancy centers in diamond, Appl. Phys. Lett. 106, 063111 (2015).

[46] Nabeel Aslam, Matthias Pfender, Rainer Stöhr, Philipp Neumann, Marc Scheffler, Hitoshi Sumiya, Hiroshi Abe, Shinobu Onoda, Takeshi Ohshima, Junichi Isoya, and Wrachtrup Jörg, Single spin optically detected magnetic resonance with 60-90 GHz (E-band) microwave resonators, Rev. Sci. Instrum. 86, 064704 (2015).

[47] Timothy J. Egan, Physico-chemical aspects of hemozoin (malaria pigment) structure and formation, J. Inorg. Biochem. 91, 19 (2002).

[48] Philippe Lavalle, Jean François Stoltz, Bernard Senger, Johannes C. Voegel, and Pierre Schaaf, Red blood cell adhesion on a solid/liquid interface, Proc. Natl. Acad. Sci. U. S. A. 93, 15136 (1996).

[49] Patrik Langehanenberg, Lyubomira Ivanova, Ingolf Bernhardt, Steffi Ketelhut, Angelika Vollmer, Dieter Dirksen, Georgi K. Georgiev, Gert von Bally, and Björn Kemper, Automated three-dimensional tracking of living cells by digital holographic microscopy, J. Biomed. Opt. 14, 014018 (2009).

[50] Janis Smits, Joshua Damron, Pauli Kehayias, Andrew F. McDowell, Nazanin Mosavian, Ilja Fescenko, Nathaniel Ristoff, Abdelghani Laraoui, Andrey Jarmola, and Victor M. Acosta, Two-dimensional nuclear magnetic resonance spectroscopy with a microfluidic diamond quantum sensor, arXiv:1901.02952 [physics.ins-det] (2019).

[51] Ghazi A. Jamjoom, Dark-field microscopy for detection of malaria in unstained blood films, J. Clin. Microbiol. 17, 717 (1983).

[52] Sooyeong Kim, Kyung Chun Kim, and Eunseop Yeom, Microfluidic method for measuring viscosity using images from smartphone, Opt. Lasers Eng. 104, 237 (2018).

[53] Renugah Naidu, Gowtham Subramanian, Ying Bena Lim, Chwee Teck Lim, and Rajesh Chandramohanadas, A reference document on permissible limits for solvents and buffers during in vitro antimalarial screening, Sci. Rep. 8, 14974 (2018).

[54] Charles Santori, Paul E. Barclay, Kai-Mei C. Fu, and Raymond G. Beausoleil, Vertical distribution of nitrogenvacancy centers in diamond formed by ion implantation and annealing, Phys. Rev. B 79, 125313 (2009).

[55] Victor M. Acosta, Andrey Jarmola, Dmitry Budker, and Lykourgos Bougas, Magnetic Resonance Spectrometer, US Patent 20180203080 A1 (2017).

[56] Shirin Attarian, Chunli Yu, Karl E. Anderson, and Ellen W. Friedman, Effects of hemin and hemodialysis in a patient with acute intermittent porphyria and renal failure, Blood Adv. 1, 915 (2017).

[57] Jennifer L. Burnett, Jennifer L. Carns, and Rebecca Richards-Kortum, In vivo microscopy of hemozoin: towards a needle free diagnostic for malaria, Biomed. Opt. Express 6, 3462 (2015).

[58] Cleofé Romagosa, Clara Menendez, Mamudo R. Ismail, Llorenç Quintó, Berta Ferrer, Pedro L. Alonso, and Jaume Ordi, Polarisation microscopy increases the sensitivity of 
hemozoin and plasmodium detection in the histological assessment of placental malaria, Acta Tropica 90, 277 (2004).

[59] Manouk Abkarian, Gladys Massiera, Laurence Berry, Magali Roques, and Catherine Braun-Breton, A novel mechanism for egress of malarial parasites from red blood cells, Blood 117, 4118 (2011).

[60] Bayden R. Wood, Antje Hermelink, Peter Lasch, Keith R. Bambery, Grant T. Webster, Mehdi Asghari Khiavi, Brian M. Cooke, Samantha Deed, Dieter Naumann, and Don McNaughton, Resonance raman microscopy in combination with partial dark-field microscopy lights up a new path in malaria diagnostics, Analyst 134, 1119 (2009).
[61] Arati G. Kolhatkar, Andrew C. Jamison, Dmitri Litvinov, Richard C. Willson, and T. Randall Lee, Tuning the magnetic properties of nanoparticles, Int. J. Mol. Sci. 14, 15977 (2013).

[62] Liam P. McGuinness, Yuling Yan, Alastair Stacey, David A. Simpson, Liam T. Hall, Dougal Maclaurin, Steven Prawer, Paul C. Mulvaney, Robert E. Scholten, and Lloyd C. L. Hollenberg, Quantum measurement and orientation tracking of fluorescent nanodiamonds inside living cells, Nat. Nanotechnol. 6, 358 (2011).

[63] Georg Kucsko, Peter C. Maurer, Norman Y. Yao, Minoru Kubo, Hyun Jong Noh, Po Kam Lo, Hongkun Park, and Mikhail Lukin, Nanometre-scale thermometry in a living cell, Nature 500, 54 (2013). 